\title{
Modeling of texture quantification and image classification for change prediction due to COVID lockdown using Skysat and Planetscope imagery
}

\author{
Amit Kumar Shakya ${ }^{1}\left[\right.$ Ayushman Ramola $^{1} \cdot$ Anurag Vidyarthi $^{2}$
}

Received: 17 May 2021 / Accepted: 9 August 2021 / Published online: 25 August 2021

(c) The Author(s), under exclusive licence to Springer Nature Switzerland AG 2021

\begin{abstract}
This research work models two methods together to provide maximum information about a study area. The quantification of image texture is performed using the "grey level co-occurrence matrix (GLCM)" technique. Image classification-based "object-based change detection (OBCD)" methods are used to visually represent the developed transformation in the study area. Pre-COVID and post-COVID (during lockdown) panchromatic images of Connaught Place, New Delhi, are investigated in this research work to develop a model for the study area. Texture classification of the study area is performed based on visual texture features for eight distances and four orientations. Six different image classification methodologies are used for mapping the study area. These methodologies are "Parallelepiped classification (PC)," "Minimum distance classification (MDC)," "Maximum likelihood classification (MLC)," "Spectral angle mapper (SAM)," "Spectral information divergence (SID)" and "Support vector machine (SVM)." GLCM calculations have provided a pattern in texture features contrast, correlation, ASM, and IDM. Maximum classification accuracy of $83.68 \%$ and $73.65 \%$ are obtained for pre-COVID and postCOVID image data through MLC classification technique. Finally, a model is presented to analyze before and after COVID images to get complete information about the study area numerically and visually.
\end{abstract}

Keywords Grey level co-occurrence matrix $\cdot$ Object-based change detection $\cdot$ Image classification $\cdot$ Texture quantification

\section{Introduction}

Estimating changes developed in land use/land cover is a hot research area these days. Researchers investigate changes in the land pattern through satellite data, microsatellite data, drone data, unmanned aerial vehicles (UAVs) data, terrain analysis, etc. (Chen et al. 2018). Several space agencies have conducted a series of successful space exploration missions like the super excited Apollo mission (Papanastassiou and Wasserburg 1971), Hubble mission (Baker et al. 2020), Voyager mission (Cohen and Rymer 2020), Cassini-Huygens mission (Sotin et al. 2021), Chandra mission (Tomsick et al.

Amit Kumar Shakya

xlamitshakya.gate2014@ieee.org

1 Department of Electronics and Communication Engineering, Sant Longowal Institute of Engineering and Technology, Sangrur, Punjab, India

2 Department of Electronics and Communication Engineering, Graphic Era (Deemed to be University), Dehradun,

Uttarakhand, India
2021) of the National Aeronautics and Space Administration (NASA). Aryabhata (Damle et al. 1976), Chandrayaan 2 carried by GSLV Mark 3 (Chandrashekar 2016), Mangalyaan (Haider and Pandya 2015), launching 104 satellites in a single attempt (Muraleedharan et al. 2019), etc., are prominent successful space missions conducted by the Indian Space Research Organization (ISRO). These space missions provide information about the capability of the individual space agency in space exploration. Due to these missions, space agencies generate extensive data to analyze specific situations or save records for future analysis (Mathieu et al. 2017). The data used in this research work is a perfect example of this scenario as the pre-COVID image of the study area was snapped for a general-purpose. Still, the postCOVID image was snapped to study the consequence of lockdown. Thus, the combination of pre-COVID data and post-COVID data becomes a great scenario to explore by remote sensing professionals, scientists, and researchers.

Nowadays, small aircraft-like devices popularly known as "drones" and UAVs are also used for data collections and day-to-day purposes (Otto et al. 2018). These devices are 
operated by the human expect or some onboard computer device (Jiang et al. 2020). They are also used for several applications related to medical diagnostic, defense, transportation, film making, scientific research, firefighting, emergency services, etc. (Kerle et al. 2020). UAVs are now introduced in satellite mapping of the land-sides affected by the landslides (Niethammer et al. 2012), crop damage assessment caused due to the natural phenomenon (Maimaitijiang et al. 2020), mapping disputed territory (defense application) (Li et al. 2020), 3D model development of terrain, etc. Today, the world is infected with the novel coronavirus (Nascimento et al. 2020) (Wang et al. 2020a, b, c). In this situation, UAV has found some new application areas like spraying the disinfectant, scanning body temperature, broadcasting the message at extremely dangerous COVID hotspots, cargo delivery, QR codes, connectivity, mapping, etc. (d'Italie 2020). Thus besides satellites, UAVs, drones, high-resolution optical cameras, etc. are some of the primary sources through which high quality imagery can be obtained. Figure 1 represents the pictorial representation of the application areas of the drones. It can be observed that in the coming future, many earth exploration activities will be performed with the assistance of drones.

In satellite, remote sensing change detection methodologies are broadly classified into two categories (Woodcock et al. 2020), i.e., "pixel-based change detection (PBCD)" and "object-based change detection (OBCD)" (Hussain et al. 2013). Pre-classification PBCD technique provide information about the study area in binary (change/no change) format. Another popular technique for image classification is OBCD through which information of study area is obtained by analysing the difference developed in the classification classes. Through OBCD techniques when pre- and post-image of an event is classified, the comparison among them is performed by analyzing the same category for both images. $\mathrm{PBCD}$ and $\mathrm{OBCD}$ methodologies are further categorized in several techniques presented in Table 1.

Some notable work done in satellite remote sensing by fusion of two algorithms, techniques, and classification schemes are presented in these reviews. Garg and Dhiman (2021) proposed a fusion of "grey level co-occurrence matrix (GLCM)" features and "local binary pattern (LBP)" to develop a novel "content based image retrieval (CBIR)" system. They have used three different classification approaches in their experiment, i.e., support vector machine (SVM), decision tree (DT) algorithm and K-nearest neighbourhood. They have concluded that their proposed algorithm performs better with superior recall, precision, and accuracy. Iqbal et al. (2021) used GLCM features fused with "machine learning (ML)" approach to obtain similarity in different crop fields. The investigation is performed on the UAV based low altitude high resolution data. They have obtained prenominal results with this merger of these two techniques. Thus the overall accuracy of their developed system is increased by $13.65 \%$. Caballero et al. (2020) obtained C band SAR imagery to differentiate between onion and sunflower crop. In their classification technique, they have used the combination of GLCM along with SVM approach. Through their developed methodology they have obtained "overall accuracy (OA)" and "Kappa coefficient $\left(K_{p}\right)$ " of $95.35 \%$ and 0.89 respectively while differentiating onion crop with sunflower crop. Singh and Singh (2020)
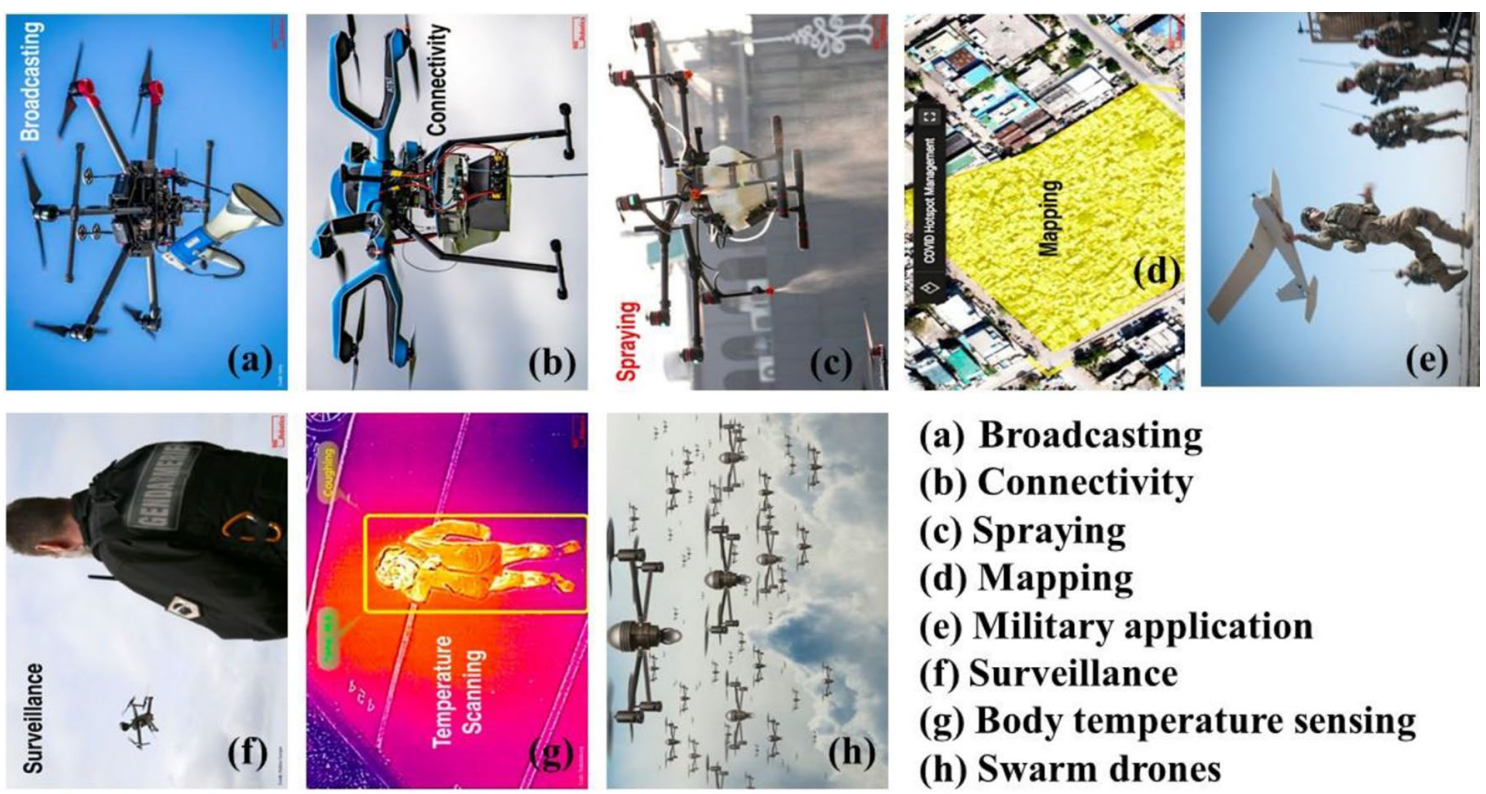

(a) Broadcasting

(b) Connectivity

(c) Spraying

(d) Mapping

(e) Military application

(f) Surveillance

(g) Body temperature sensing

(h) Swarm drones

Fig. 1 Application areas of drones (d'Italie 2020) 
Table 1 Classification of various PBCD and OBCD techniques

\begin{tabular}{|c|c|c|}
\hline \multicolumn{3}{|l|}{$\mathrm{PBCD}$ and $\mathrm{OBCD}$ change detection techniques } \\
\hline \multirow{2}{*}{$\begin{array}{l}\text { Pre classification change detection (binary } \\
\text { information about changes) } \\
\text { PBCD techniques }\end{array}$} & \multicolumn{2}{|c|}{ Post classification change detection (detail information about changes) } \\
\hline & PBCD techniques & OBCD techniques \\
\hline $\begin{array}{l}\text { Image differencing (ID) (Seydi et al. 2020; } \\
\text { Arefin et al. 2020) }\end{array}$ & $\begin{array}{l}\text { Composite or multi-date classification (Venu- } \\
\text { gopal 2020) }\end{array}$ & $\begin{array}{l}\text { Direct object change detection (DOCD) (Saha } \\
\text { et al. 2021) }\end{array}$ \\
\hline $\begin{array}{l}\text { Image ratio (IR) (Zhu et al. 2020; Kalaiselvi } \\
\text { and Gomathi 2020) }\end{array}$ & Machine learning (Pati et al. 2020) & $\begin{array}{l}\text { Classified object change detection (COCD) (Shi } \\
\text { et al. 2020) }\end{array}$ \\
\hline $\begin{array}{l}\text { Image regression (IReg) (Zhao et al. 2021; } \\
\text { Zhang et al. 2020) }\end{array}$ & GIS-based (Wang et al. 2020a, b, c) & $\begin{array}{l}\text { Multi-temporal object change detection } \\
\text { (MOCD) (Eid et al. 2020) }\end{array}$ \\
\hline $\begin{array}{l}\text { Vegetation index differencing (VID) (Polykre- } \\
\text { tis et al. 2020) }\end{array}$ & Texture analysis (Hajeb et al. 2020) & \\
\hline $\begin{array}{l}\text { Change vector analysis (CVA) (Du et al. } \\
\text { 2020) }\end{array}$ & Fuzzy change detection (Liu et al. 2020) & \\
\hline \multirow[t]{2}{*}{$\begin{array}{l}\text { Principle component analysis (PCA) } \\
\text { (Schwartz et al. 2020) }\end{array}$} & $\begin{array}{l}\text { Multi-sensor data fusion (Wang et al. 2020a, } \\
\text { b, c) }\end{array}$ & \\
\hline & Deep learning (Khelifi and Mignotte 2020) & \\
\hline
\end{tabular}

used SCATSAT-1 data to distinguish "multi-year ice" and "first-year ice" of the arctic region using "maximum likelihood classification (MLC)". They have obtained over all classification accuracy of $92 \%$ in their experiment. Rimal et al. (2020) used the Landsat imagery of the Kathmandu valley of Nepal between 1988 and 2016 to compare the efficiency of object-based image classification "SVM" and "ML" image classification algorithm. The experimental result obtained from their investigation suggest that SVM performs better than ML classification algorithm. Thus scientist and researchers are working to develop a new methodology by combining two or more techniques to obtain maximum accuracy and complete information from image classification and feature quantification.

In this research work, a model is presented employing a combination of PBCD (texture analysis based GLCM) and OBCD (Classified object change detection COCD) techniques by analyzing the pre-COVID and post-COVID (during lockdown) panchromatic images of Connaught Place, New Delhi, India. The pixel-based texture analysis GLCM technique is used for texture classification and quantification of the study area. GLCM, has provided information about the statistical and spectral behavior of the image pixels through the mathematical analysis. The quantification of the GLCM features for pre-COVID and post-COVID images produces a new relationship among GLCM features. Histogram signature plotting represents the changes in the frequency of intensity values of the study area. OBCD technique provides information about the study area in a different pattern. In this technique, for pre-COVID and post-COVID images, "region of interest (ROI)" is selected by allotting pixels values to the ROIs. These ROI behave as a "region" based on which classification of the study area is performed. Another set of ROI is also created, which assists in the accuracy assessment. The advantage of OBCD technique over PBCD technique lies in the "visual point of view." In this classification, there is also the possibility to compare only a "particular class," leaving rest classes.

The article is divided into six separate sections. "Background of PBCD (GLCM) and OBCD techniques" provides detailed background information about PBCD and OBCD techniques. "Background of Skysat satellite program and details of the study area" presents a brief report on the Skysat satellite program and study area. "Experimental results" offers detail regarding experimental results of texture quantification and image classification. "Discussion" presents discussions and outcomes from the proposed research work. Finally, "Conclusion" offers concluding remarks on the research work.

\section{Background of PBCD (GLCM) and OBCD techniques}

\section{GLCM-based texture classification technique}

The texture is an essential aspect of gathering information from remote sensing images. Through texture analysis, spectral as well as spatial information of the study area is obtained. This technique is extensively used in various inaccessible sensing applications. Harlick et al. invented GLCM (1973). He presented a set of "fourteen" different features to classify the image texture (Harlick et al 1973). Later a lot of work was done on these features. Gotlieb and Kreyszig organized these fourteen features into a set of four different categories (1990). Visual texture features are considered 


\begin{tabular}{|c|c|c|c|c|c|}
\hline$(i, j)$ & 1 & 2 & 3 & 4 & 5 \\
\hline 1 & $(1,1)$ & $(1,2)$ & $(1,3)$ & $(1,4)$ & $(1,5)$ \\
\hline 2 & $(2,1)$ & $(2,2)$ & $(2,3)$ & $(2,4)$ & $(2,5)$ \\
\hline 3 & $(3,1)$ & $(3,2)$ & $(3,3)$ & $(3,4)$ & $(3,5)$ \\
\hline 4 & $(4,1)$ & $(4,2)$ & $(4,3)$ & $(4,4)$ & $(4,5)$ \\
\hline 5 & $(5,1)$ & $(5,2)$ & $(5,3)$ & $(5,4)$ & $(5,5)$ \\
\hline
\end{tabular}

(a)

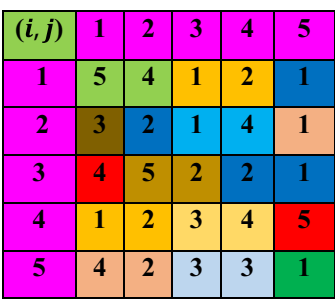

(b)

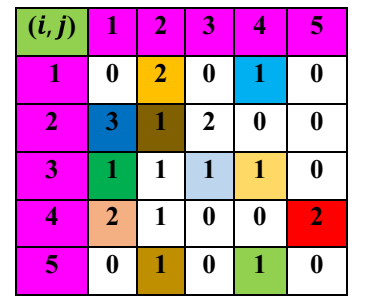

(c)

\begin{tabular}{|c|c|c|c|c|c|}
\hline$(i, j)$ & 1 & 2 & 3 & 4 & 5 \\
\hline 1 & $0 / 25$ & $2 / 25$ & $0 / 25$ & $1 / 25$ & $0 / 25$ \\
\hline 2 & $3 / 25$ & $1 / 25$ & $2 / 25$ & $0 / 25$ & $0 / 25$ \\
\hline 3 & $1 / 25$ & $1 / 25$ & $1 / 25$ & $1 / 25$ & $0 / 25$ \\
\hline 4 & $2 / 25$ & $1 / 25$ & $0 / 25$ & $0 / 25$ & $2 / 25$ \\
\hline 5 & $0 / 25$ & $1 / 25$ & $0 / 25$ & $1 / 25$ & $0 / 25$ \\
\hline
\end{tabular}

(d)
Fig. 2 a Pixel position of the input image, $\mathbf{b}$ input image of dimension $5 \times 5$, $\mathbf{c}$ GLCM of the input image, and $\mathbf{d}$ normalized representation of the GLCM image

most important in remote sensing applications because they directly impact human visual perception. Texture visual features include contrast, correlation, angular second moment (ASM), and inverse difference moment (IDM) (Haralick et al. 1973). The GLCM formation from an input image is presented in Fig. 2. The location of pixel position in the input image is illustrated in Fig. 2a. Input image of dimension $5 \times 5$ is presented in Fig. 2 b. The GLCM image of the input image is shown in Fig. 2c. The normalized GLCM image is presented in Fig. 2 d.

GLCM calculation of any input image is dependent on two critical parameters. These parameters are "distance" and "angle of orientation." The distance represents space between the "pixel of interest" and the "neighboring pixel." This distance can be varied to obtain different values of the texture features. The distance can be varied starting from $d=1, d=2$ and so on. The orientation angle presents the direction of the variation of the texture features following the distance. The orientation angle of an image can vary from $0^{\circ}$ to $315^{\circ}$. This situation can be understood from Fig. 3, where different combinations of distances and orientations from a "pixel of interest (POI)" are presented.

Let us assume an image with $N_{x}$ resolution cells in the "horizontal direction" and $N_{y}$ resolution cells in the "vertical direction." The grey tone shown up in the image is quantized to $N_{g}$ level. Then the "horizontal spatial domain," "vertical spatial domain," and the "set of the quantized grey levels" are expressed by $l_{x}, l_{y}$ and $G$. The set $l_{x} \times l_{y}$ is the resolution cell. Thus the grey tone in each resolution cell is expressed as $l_{x} \times l_{y}: G$. Therefore the expression for the angle quantized from $0^{\circ}$ to $315^{\circ}$ are expressed by Eqs. (1)-(8), where $(k, l)$ represents the image pixels.

$\operatorname{pix}\left(k, l, \operatorname{Dis}, 0^{\circ}\right)=\neq\left\{\left(((m, n),(o, p)) \in\left(l_{x} \times l_{y}\right)\right) \times\left(l_{x} \times l_{y}\right) \vdots|m-n|=0,|o-p|=D\right\}$,

$I(m, n)=k$ and $I(o, p)=l$

$\operatorname{pix}\left(k, l, \operatorname{Dis}, 45^{\circ}\right)=\neq\left\{\left(((m, n),(o, p)) \in\left(l_{x} \times l_{y}\right)\right) \times\left(l_{x} \times l_{y}\right) \vdots|m-n|=-D,|o-p|=D\right\}$,

$I(m, n)=k$ and $I(o, p)=l$

$\operatorname{pix}\left(k, l, \operatorname{Dis}, 90^{\circ}\right)=\neq\left\{\left(((m, n),(o, p)) \in\left(l_{x} \times l_{y}\right)\right) \times\left(l_{x} \times l_{y}\right) \vdots|m-n|=-D,|o-p|=0\right\}$,

$I(m, n)=i$ and $I(o, p)=j$

Fig. 3 GLCM pixel, distance, and the orientation arrangement from the center image pixel

\section{Pixels, distance and orientation arrangement}

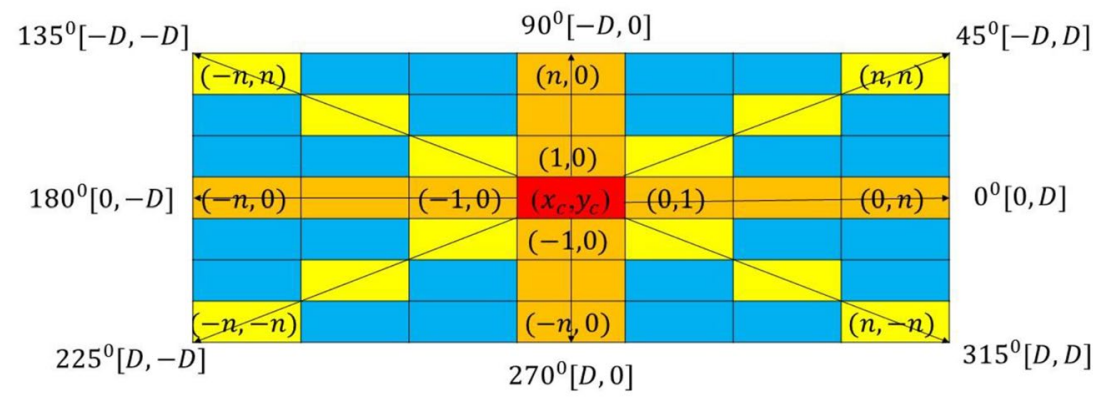

Spatial relationship between pixels and array of offsets from the central pixel 
$\operatorname{pix}\left(k, l, \operatorname{Dis}, 135^{\circ}\right)=\neq\left\{\left(((m, n),(o, p)) \in\left(l_{x} \times l_{y}\right)\right) \times\left(l_{x} \times l_{y}\right) \vdots|m-n|=-D,|o-p|=-D\right\}$,

$I(m, n)=i$ and $I(o, p)=j$

$\operatorname{pix}\left(k, l, \operatorname{Dis}, 180^{\circ}\right)=\neq\left\{\left(((m, n),(o, p)) \in\left(l_{x} \times l_{y}\right)\right) \times\left(l_{x} \times l_{y}\right) \vdots|m-n|=0,|o-p|=-D\right\}$,

$I(m, n)=i$ and $I(o, p)=j$

$\operatorname{pix}\left(k, l, \operatorname{Dis}, 225^{\circ}\right)=\neq\left\{\left(((m, n),(o, p)) \in\left(l_{x} \times l_{y}\right)\right) \times\left(l_{x} \times l_{y}\right) \vdots|m-n|=D,|o-p|=-D\right\}$,

$I(m, n)=i$ and $I(o, p)=j$

$\operatorname{pix}\left(k, l, \operatorname{Dis}, 270^{\circ}\right)=\neq\left\{\left(((m, n),(o, p)) \in\left(l_{x} \times l_{y}\right)\right) \times\left(l_{x} \times l_{y}\right) \vdots|m-n|=D,|o-p|=0\right\}$,

$I(m, n)=i$ and $I(o, p)=j$

$\operatorname{pix}\left(k, l, \operatorname{Dis}, 315^{\circ}\right)=\neq\left\{\left(((m, n),(o, p)) \in\left(l_{x} \times l_{y}\right)\right) \times\left(l_{x} \times l_{y}\right) \vdots|m-n|=D,|o-p|=D\right\}$,

$I(m, n)=i$ and $I(o, p)=j$

Texture features developed by Haralick other than visual texture features are presented in Eqs. (9)-(18) (1973). These features are based on information theory, statistical measures, and information measures of correlation.

GLCM features based on the "information theory," in particular, entropy

1. Sum entropy $=-\sum_{k=2}^{2 N_{g}} p_{(x+y)}(k) \log \left\{p_{x+y}(k)\right\}$

2. $\quad$ Entropy $=-\sum_{k} \sum_{l} p(k, l) \log \{p(k, l)\}$

3. Difference entropy $=-\sum_{k=0}^{N_{g}-1} p_{(x-y)}(k) \log \left\{p_{(x-y)}(k)\right\}$

Where $x$ and $y$ denote elements of the "row" and "column" respectively of the co-occurrence matrix and $p_{(x+y)}$ represents the "probability of the co-occurrence matrix" corresponding to $x+y$. Similarly $p_{(x-y)}$ represent the "probability of the co-occurrence matrix" corresponding to $x-y$.

GLCM features based on "statistical measures."

4. Inverse difference moment $=\sum_{k} \sum_{l} \frac{1}{1+(k-l)^{2}} p(k, l)$
5. Sum average $=\sum_{k=2}^{2 N_{g}} k \times p_{(x+y)}(k)$

6. Sum variance $=\sum_{k=2}^{2 N_{g}}\left(k-f_{s}\right)^{2} p_{(x+y)}(k)$

7. Difference variance $=\sum_{k=0}^{N_{g}-1} k^{2} \times p_{(x-y)}(k)$

GLCM features based on the "information measure of correlation."

8. Information measures of Correlation $1=\frac{\mathrm{HXY}-\mathrm{HXY} 1}{\operatorname{MAX}\{\mathrm{HX}, \mathrm{HY}\}}$

9. Information measures of Correlation 2

$$
=(1-\exp [-2(\mathrm{HXY} 2-\mathrm{HXY})])^{1 / 2}
$$

Where HXY $=-\sum_{k} \sum_{l} p(k, l) \log (p(k, l)), \mathrm{HX}, \mathrm{HY}$ are the entropies of $p_{x}$ and $p_{y}, \mathrm{HXY} 1=-\sum_{k} \sum_{l} p(k, l) \log \left\{p_{x}(k) p_{y}(l)\right\}$, $\mathrm{HXY} 2=-\sum_{k} \sum_{l} p_{x}(k) p_{y}(l) \log \left\{p_{x}(k) p_{y}(l)\right\}$ 
Table 2 Discussion of the visual texture features

\begin{tabular}{|c|c|c|c|}
\hline S. no & Texture features & Mathematical expression & Discussion \\
\hline 1 & IDM & $f(k, l)=\sum_{k} \sum_{l} \frac{1}{1+(k-l)^{2}} p(k, l)$ & $\begin{array}{l}\text { This parameter measures the closeness of the distribution between a pixel } \\
\text { of interest and neighboring pixels. It is the measure of the similarity of } \\
\text { the GLCM element along the GLCM diagonal. The normalized range of } \\
\text { homogeneity is }[0,1]\end{array}$ \\
\hline 2 & ASM & $f(k, l)=\sum_{k} \sum_{l}\{p(k, l)\}^{2}$ & $\begin{array}{l}\text { It is a measure of the sum of squared elements in the GLCM. This parameter } \\
\text { is also known as "energy." The normalized range of "energy" is }[0,1]\end{array}$ \\
\hline 3 & Correlation & $f(k, l)=\frac{(k, l) p(k, l)-\mu_{k} \mu_{l}}{\sigma_{k} \sigma_{l}}$ & $\begin{array}{l}\text { It is the measure of the "joint probability occurrence" of the specified pixel } \\
\text { pairs. The normalized range of the correlation is }[-1,1] \text {. But good correla- } \\
\text { tion value lies in the positive direction }\end{array}$ \\
\hline 4 & Contrast & $f(k, l)=\sum_{0}^{n_{g}-1} n^{2} \times\left\{\sum_{k=1}^{n_{g}} \sum_{l=1}^{n_{g}} p(k, l)\right\}$ & $\begin{array}{l}\text { It is the measure of the local variations in the GLCM. The normalized } \\
\text { range of contrast is }[0,1] \text {. If the value of the discrepancy is zero, then it is } \\
\text { a constant image. } n_{g} \text { is the number of "distinct grey levels" in the image. } \\
\text { The difference in the grey level is the }|k-l| \text {. If the }|k-l| \text { is a zero, then the } \\
\text { image is a constant image with no variation }\end{array}$ \\
\hline
\end{tabular}

10. Maximum Correlation Coefficent $=(\text { second }- \text { largest eigenvalue of } Q)^{0.5}$ here $Q(k, l)=\sum_{k} \frac{p(k, l) p(k, l)}{p_{x}(j) p_{y}(k)}$

GLCM features representing visual texture features are presented in Table 2, which explains their mathematical notation, range, and discussion of these features.

The pixel-based GLCM techniques have several advantages and shortcomings listed as follows.

\section{Advantages of GLCM}

In the GLCM based change detection technique, spectral and spatial information of the study area is obtained.

GLCM offers two different procedures for the quantification of the image pixels. Firstly selection of the window size of dimensions like $3 \times 3$ or $5 \times 5$ over a complete image. Secondly, numerical quantification of the total image pixels can be performed, and on that basis, texture features can be quantified.

GLCM can offer information about the image features in four categories based on human visual perception, statistical measures, entropy measures, and correlation information.

The future aspect of the GLCM can be understood from the fact that earlier GLCM can provide information only in two dimensional (2D) surfaces. Today, researchers and scientists have developed a procedure to calculate the GLCM across three dimensions known as 3D GLCM. GLCM can calculate the pixel brightness of the image through different combinations of the image pixels.

GLCM is used in remote sensing applications, but today, the GLCM technique is also used in earth scattering data analysis to predict "Earthquakes" and "Tsunami" possibilities.

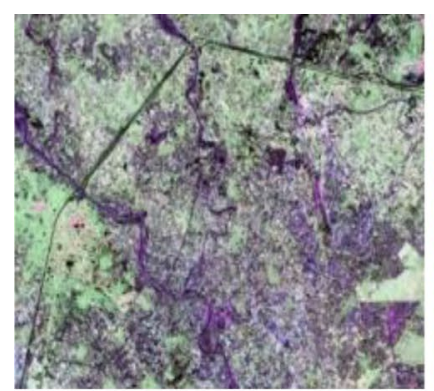

(a)

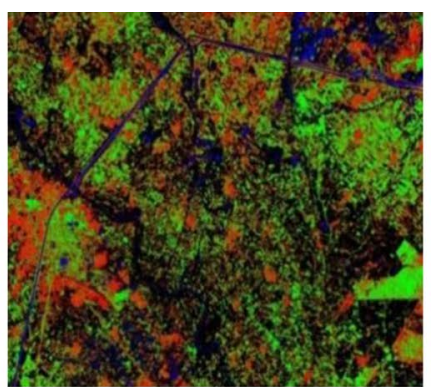

(b)

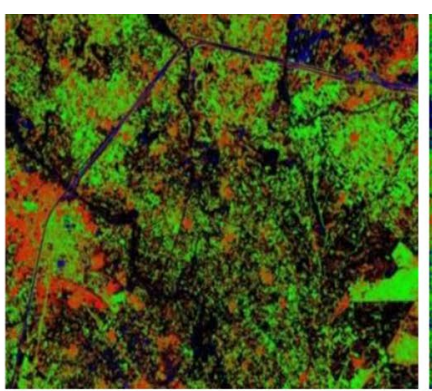

(c)

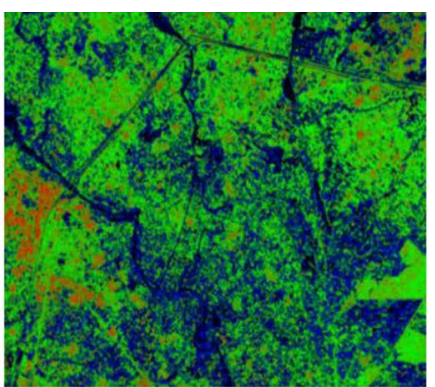

(d)

Fig. 4 a Band fused PALSAR data, b classified image with four prominent class, $\mathbf{c}$ classified image with three prominent class, and $\mathbf{d}$ classified image with two prominent class (Data Courtesy: Japanese Aerospace Exploration Agency, JAXA) 
An essential advantage of the GLCM is that its features can be obtained for the "single orientation and distance" along with "combination of directions and distances."

\section{Shortcoming of GLCM}

Computation of the GLCM is a time-consuming process. The main problem during GLCM calculation is the computational cost using pixel to pixel combination of the image. The issue of GLCM computation can be overcome by the use of GLCM with the Sobel operator.

\section{Background of the image classification technique}

Image classification techniques are used to classify the image into several small objects or classes. These objects can be classified as soil, urban, agriculture, plants, trees, water, etc. When an image is segregated using an image classification technique, all the essential areas of the image can be classified into objects or classes. These areas of the image selected as the object depend upon the type of study, e.g., Fig. 4a consists of a band-fused "Phased array $L$ band synthetic aperture radar (PALSAR)" image of the Roorkee region of Uttarakhand, India. This image is classified using three different classification techniques and in four other classes, i.e., bare soil (black color), water (blue color), urban (red color), and agriculture (green color). Thus if anyone wishes to study only two classes, water, and agriculture, their task is completed by Fig. 4d. If a study about three categories is required, they can opt for Fig. $4 \mathrm{c}$ and can obtain information about four classes through Fig. 4b. Thus performing an image classification and creating the number of objects depends entirely upon the application.

A comparison can be made when pre and post-image related to any natural phenomenon are classified based on several classes. Then this type of image classification approach falls under object-based change detection (OBCD)
(Zhang et al. 2018). When the comparison is made between two images using OBCD methodologies, they are compared based on standard classification features, i.e., "user accuracy (UA) (Tong and Feng 2020)", "producer accuracy (PA) (Tong and Feng 2020)", "commission error (CE) (Agariga et al. 2021)," "omission error (OE) (Agariga et al. 2021)," "overall accuracy (OA) (Tong and Feng 2020)", and "kappa coefficient $\left(K_{p}\right)$ (Tong and Feng 2020)." Some of the prominent image classification techniques using object formation to classify an image are "Maximum likelihood classification (MLC)" (Soni et al. 2021), "Spectral angle mapper (SAM)" (Wang et al. 2021), "Support vector machine (SVM)" (Leonga et al. 2021), "Minimum distance classification (MDC)" (Nie et al. 2021), "Parallelepiped classification (PC)" (Kundu et al. 2021), and "Spectral information divergence (SID)" (Hunt 2021). Brief details of these classification techniques and their methodologies are presented in these reviews.

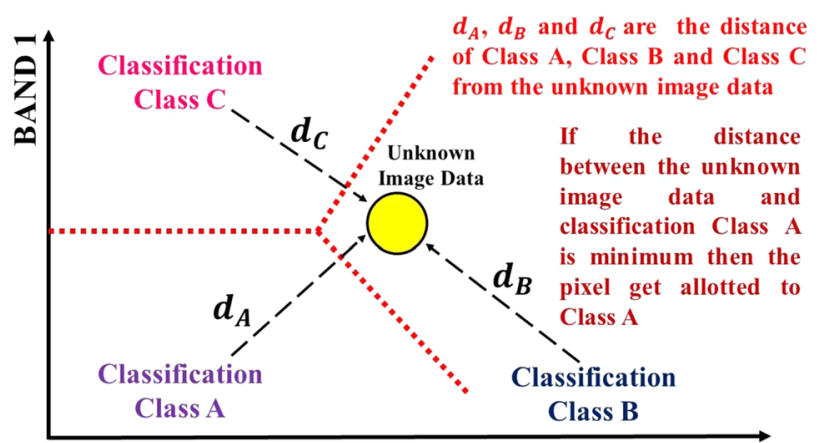

BAND 2

Fig. 6 Unknown data allotment to a particular class
Fig. 5 Pixel allotment to a particular class according to mean value

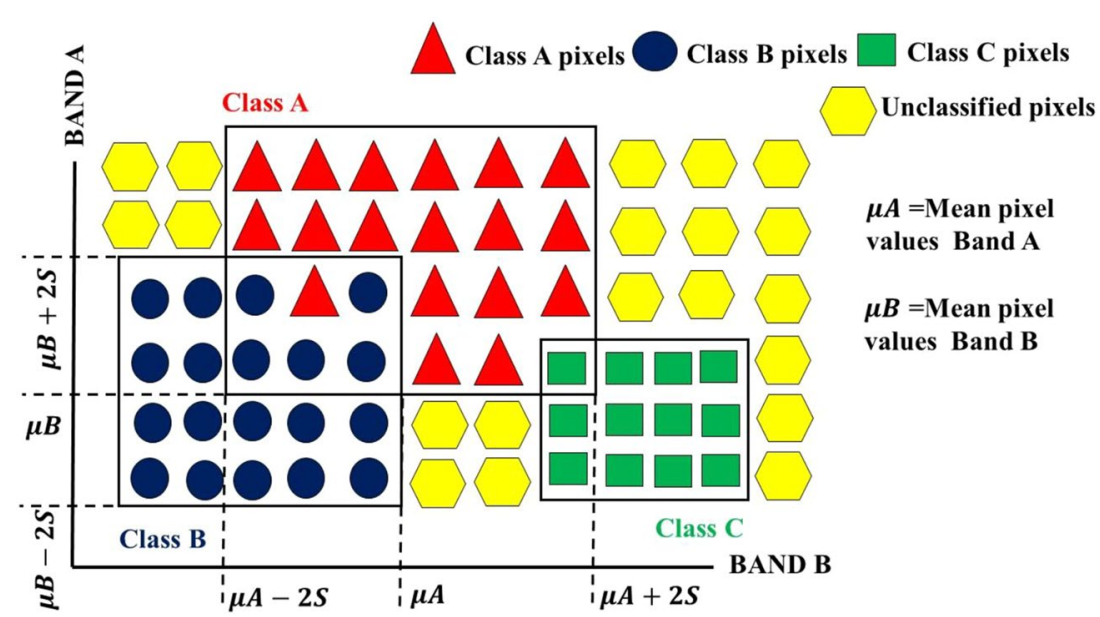




\section{Parallelepiped classification (PC)}

PC uses a "decision rule" to classify the multispectral, hyperspectral, RGB image data. The image data boundaries are created through the " $n$-dimensional parallelepiped" in the image data space. Figure 5 represents the classification of the image data through parallelepiped classification. Here image pixels are classified into four different classes, i.e., Class A, Class B, Class C, and unclassified pixels. Here, while performing these classifications, pixels of one class get merged with the other class's pixel unintentionally. The user estimates the minimum and maximum pixel value corresponding to each band or a range in this classification scheme. It is expressed in terms of "standard deviation" on either side of the "mean" of each feature. These values determine the scope of the parallelepiped classification, i.e., for Band $\mathrm{A}$, the range of the category is $\mu A-2 S \leq P C \leq \mu A+2 S$, and the content of variety for Band B is $\mu B-2 S \leq P C \leq \mu B+2 S$ where $S$ is the standard deviation of the image.

\section{Advantage of parallelepiped classification}

- This technique performs fast image classification.

- This technique is suitable for non-normal distribution.

- This technique can be applied over a limited land cover area.

\section{Shortcoming of parallelepiped classification}

- Overlapping of classified classes are allowed in this classification technique, resulting in less accurate results.

- In this classification technique, all the pixels are not classified.

- One of the main problems with this algorithm is that pixels are spectrally apart from the signature mean, affecting the pixel classification.

\section{Minimum distance classification (MDC)}

MDC technique is employed to classify "unknown image data" into separate object classes presented in Fig. 6. This approach's main objective is to minimize the distance between the different object classes and anonymous image data. In other words, the distance is considered as the parameter of similarity, so the minimum distance between two observation classes is identical to the maximum similarity. The minimum distance can be calculated with the assistance of Eq. (19).

$\left.d_{x_{t p} \mu_{c}}=\left(\sqrt{\left(x_{t p}^{2}\right.}-\mu_{c}^{2}\right)\right)$

where $\left(x_{t p}\right)$ is defined as the test pixel and $\left(\mu_{c}\right)$ represents the mean value of the classified object.

\section{Advantage of minimum distance classification}

- All the regions of the $n$-dimensional space get classified under this classification scheme.

- The main advantage of this classification scheme is no overlapping of the image classes occurs during the image classification.

\section{Shortcoming of minimum distance classification}

- In this classification scheme, spectral variability is assumed to be the same in all directions, producing false results.
Fig. 7 Pixel allotment in "Maximum likelihood image classification"

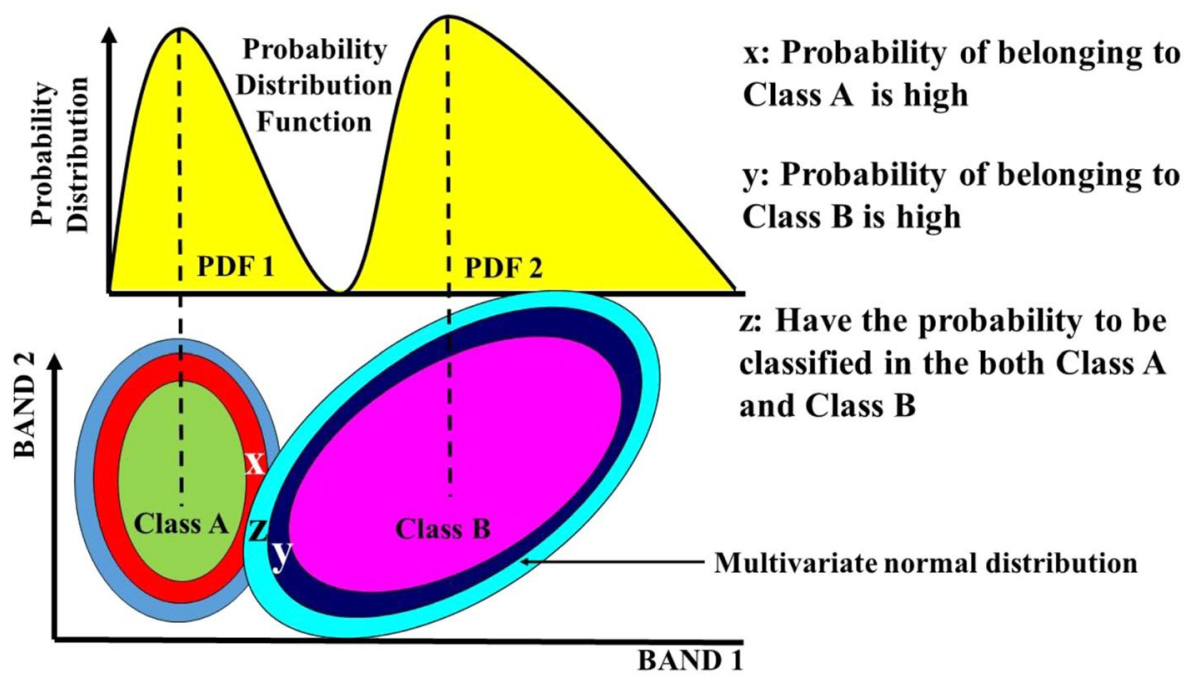




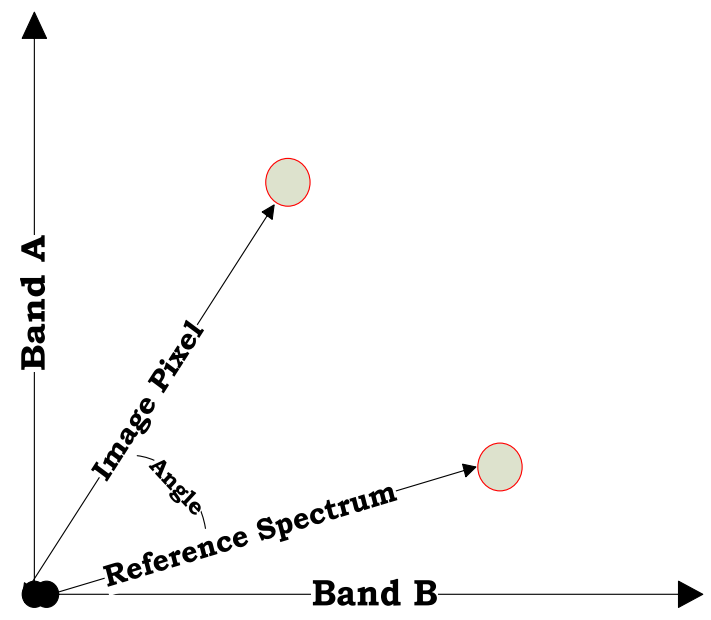

Fig. 8 Representation of the spectral angle mapper corresponding to "Band A" and "Band B"

\section{Maximum likelihood classification (MLC)}

This classification technique assumes that an individual class's statistics in the respective band are typically distributed, represented in Fig. 7. This methodology calculates the "probability of the particular pixel" to a specific category, and that pixel is assigned to the object class having the "maximum probability" or "maximum likelihood."

The mathematical relationship for establishing the maximum likelihood between the image pixels is expressed by Eq. (20).

$L_{p}(x)=\frac{1}{(2 \pi)^{n / 2} \sum k^{\frac{1}{2}}} \exp \left[-\frac{1}{2\left(x-\mu_{p}\right) \sum_{p}^{-1}\left(x-\mu_{p}\right)^{-1}}\right]$

where $x$ represents the $n$ band image data, $L_{p}(x)$ is the expression of the "likelihood of object" of class $x$ belonging to the class $p . \mu_{p}$ represents the "mean vector" of the class $k . \sum p$ is the representation for the "variance matrix."

\section{Advantage of maximum likelihood classification}

- This scheme is assumed to be the most sophisticated as under this image classification scheme, good separation between different classes is obtained.

\section{Shortcoming of maximum likelihood classification}

- Accuracy assessment requires an intense training of the dataset to describe covariance and the mean structure of the classes.

\section{Spectral angle mapper (SAM)}

The created object's spectrum is compared with the already known "object spectrum" in this classification technique." As a result of SAM classification, an image is obtained with the best match for the individual image pixel. This similarity in the range is analyzed in terms of similarity, with the "vector originated from the origin." Usually, reflection intensity is represented by the length of the vectors illustrated in Fig. 8.

The spectrum angle describes the difference between the spectra of Band A and Band B. Finally, an image is classified into several classes by evaluating the rise developed between the reference spectrum and the object's spectrum. The angle formed between two vectors is the cosine angle. This angle is expressed by Eq. (21).

$\alpha=\cos ^{-1}\left[\frac{\sum_{k=1}^{l=\mathrm{nb}} t_{k} \mathrm{rp}_{k}}{\left(\sum_{k=1}^{l=\mathrm{tb}} t_{k}^{2}\right)^{1 / 2}\left(\sum_{k=1}^{l=\mathrm{tb}} \mathrm{rp}_{k}^{2}\right)^{1 / 2}}\right]$

where $\alpha$ (alpha) is the angle between the "two vectors," nb represents the "total numbers of the spectral bands," $t_{i}$ is the "target pixels" present in an image, and rp is the total number of the reference pixels.

\section{Advantage of spectral angle mapper}

- This method is considered a user-friendly and quick method to map the spectral similarity of the image spectra against a reference spectrum.

- This technique produces good classification results even in the presence of the scaling noise.

\section{Shortcoming of spectral angle mapper}

- This technique does not respond to a situation in which a vector magnitude fails to discriminate information in many instances.

\section{Spectral information divergence (SID)}

SID uses a "spectral classification approach" to compare image pixels with the reference spectrum. The tool used for the comparison is the divergence measure. Slight divergence is the indication of the small image pixels. Pixels having considerable divergence values above a predefined threshold are not classified under this approach. Spectra of the end member can be extracted directly from an image. The SID method computes the "spectral similarity" based on the divergence between the probability distribution of two 
Fig. 9 Band fusion in the spectral information divergence

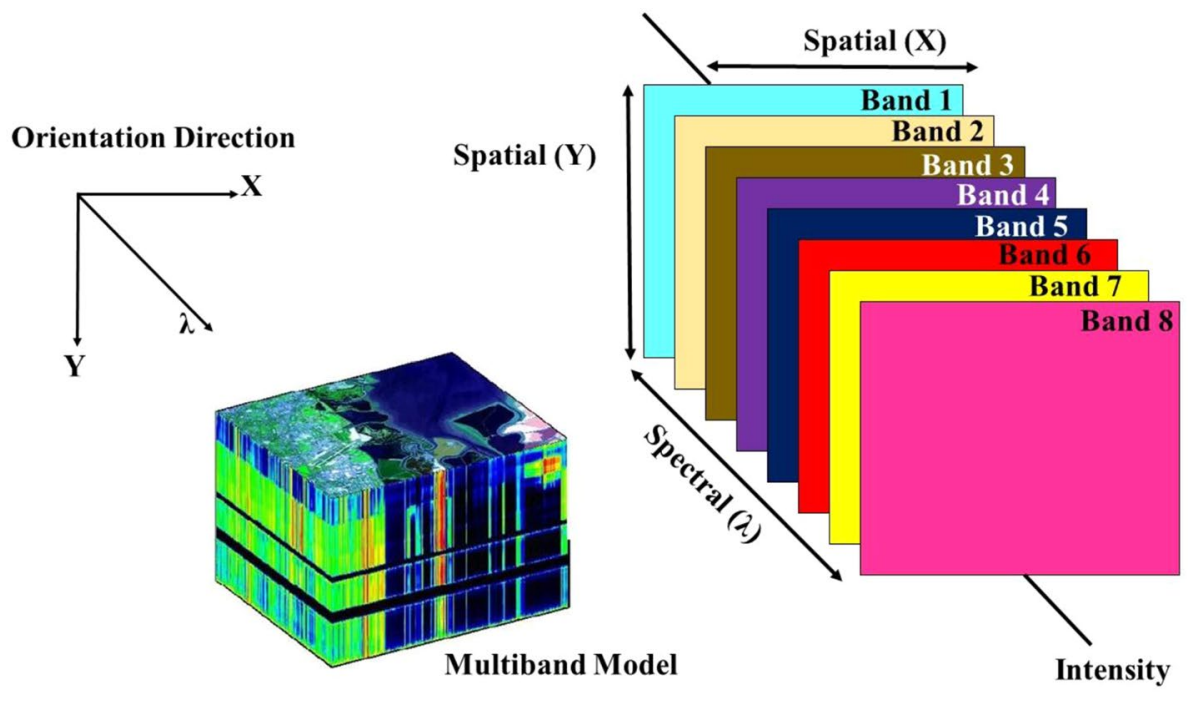

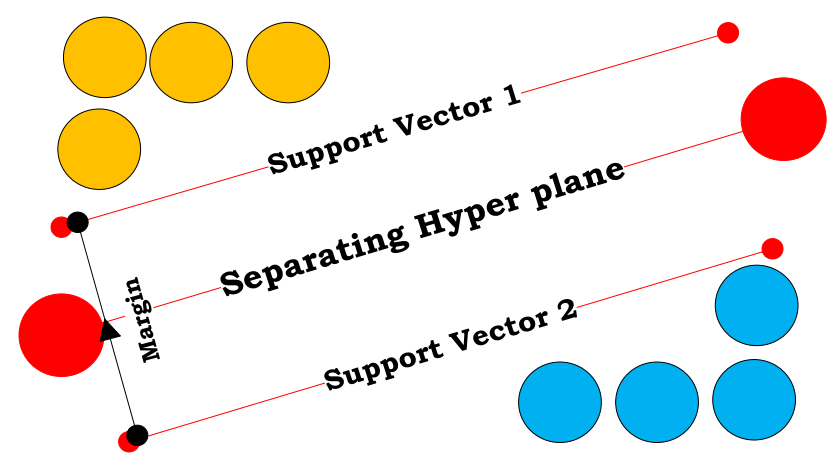

Fig. 10 Hyperloop diagram for support vector machine

spectra. Let us assume an embodiment with reference spectra $(R)$ and test spectra $(T)$.

The distribution value for the reference spectra can be expressed by Eq. (22).

$d_{\mathrm{vr}}=\frac{R_{k}}{\sum_{k=1}^{C} R_{k}}$

The distribution value for the test spectra can be expressed by Eq. (23).

$d_{\mathrm{vt}}=\frac{T_{k}}{\sum_{k=1}^{C} T_{k}}$

The SID corresponding to the reference and the test spectra is expressed by Eq. (24).

$\mathrm{SID}=\sum_{k=1}^{C} d_{\mathrm{vt}} \log \left(\frac{d_{\mathrm{vt}}}{d_{\mathrm{vr}}}\right)+\sum_{k=1}^{C} d_{\mathrm{vr}} \log \left(\frac{d_{v r}}{d_{v t}}\right)$
Figure 9 represents the stacking of bands from Band 1 to Band 8 to develop a multiband image model using the SID technique.

\section{Advantage of spectral information divergence}

- SID measures the amount of deviation by analyzing the probabilistic behaviors of the pixel's spectral signatures. This comparison depends on the information theory, which is considered the more effective in retaining spectral properties; through this methodology, the spectral similarity between two image pixels can also be measured.

\section{Shortcoming of spectral information divergence}

- SID is considered an efficient image processing technique. A critical drawback of this technique is the variation in the "output results" due to a change in the "light intensity," which affects the classification results.

\section{Support vector machine (SVM)}

The "support vector machine (SVM)" classification technique is based on "supervised learning for data analysis and study." This technique uses a "machine learning approach." This technique follows image classification and regression data analysis. SVM follows the kernel principle to perform linear and nonlinear image regression. In this algorithm, two hyperplanes are separated from each other using "support vector 1" and "support vector 2". The pixels that need to be classified are situated opposite the hyperplane represented by Fig. 10. This algorithm of image classification was developed by "Hava Siegelmann" and "Vladimir Vapnik." (Tiwari et al. 2021). This algorithm was initially developed 
for computer vision and pattern recognition but later used in satellite remote sensing and image processing applications.

The hypothesis function is expressed by Eq. (25).

$H\left(L_{x}\right)=\left\{\begin{array}{l}+1 \text { ifz } x+l \geq 0 \\ -1 \text { ifz } x+l<0\end{array}\right\}$

Thus the pixels "above the hyperplane" is classified as +1 , and the pixels "below the hyperplane" is classified as -1 .

\section{Advantage of the SVM}

- Perform effective classification in high dimensions compared to the $K$ nearest neighbor algorithm.

- The most effective classification technique for the cases having several sizes and remarkable than the number of samples.

- SVM is a versatile technique having different functions to be specified for the decision function.

- When the separation margin between a plane is evident, then this methodology works most efficiently.

\section{Shortcoming of SVM}

- Several parameters like Kernel, $C$-, and Gamma function need to be set correctly in the SVM classification approach to obtain better classification results. So at the same time, different parameters need to be taken care of for better classification.
- SVM does not assist in determining probability estimates directly. These features require a "fivefold cross-validation process" to compute.

- A good classification result is challenging to obtain through this methodology when the dataset contains extreme noise.

\section{Background of Skysat satellite program and details of the study area}

In today's world, several states governments, and private industries compete to explore massive and vital information from Earth. Several government space agencies are actively participating in researching information about the origin of a novel coronavirus and its impact on the daily activity of ordinary human beings. Currently, six different space agencies "European Space Agency (ESA) (Wörner 1975)," "National Aeronautics and Space Administration (NASA) (Dunbar 1958)," "Japan Aerospace Exploration Agency (JAXA) (Yamakawa 2003)," "Russian Federal Space Agency (RFSA or Roscosmos) (Government 1992)," "China National Space Administration (CNSA) (Kejian 1993)" and "Indian Space Research Organisation (ISRO) (Sarabhai 1969)" are working in the field of satellite launch and satellite recovery. These agencies have their own satellite launch capacities. Besides these their several other governments sponsored space agencies like the "Canadian Space Agency (François-Philippe 1989)," "UK Space Agency (Annett 2010)," "Australian

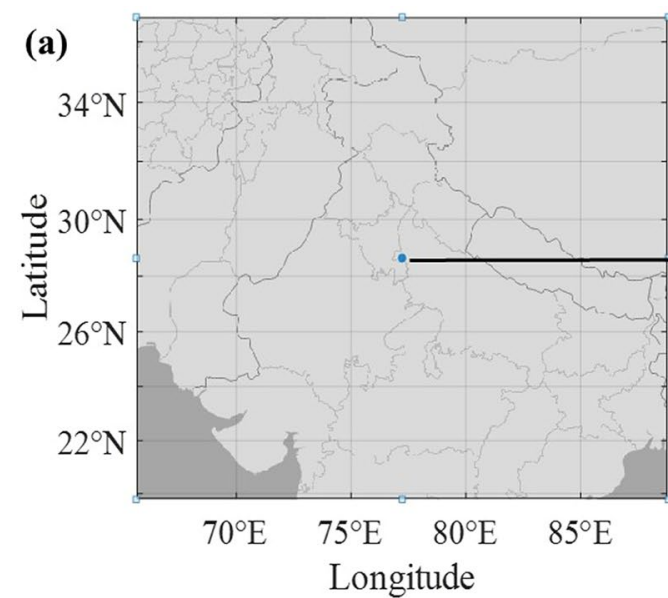

(a) India (Matlab)

(b) New Delhi (Google Earth)

(c) Connaught Place (Google Earth)

(d) Connaught Place Pre Covid (Skysat)

(e) Connaught Place Post Covid (Skysat)
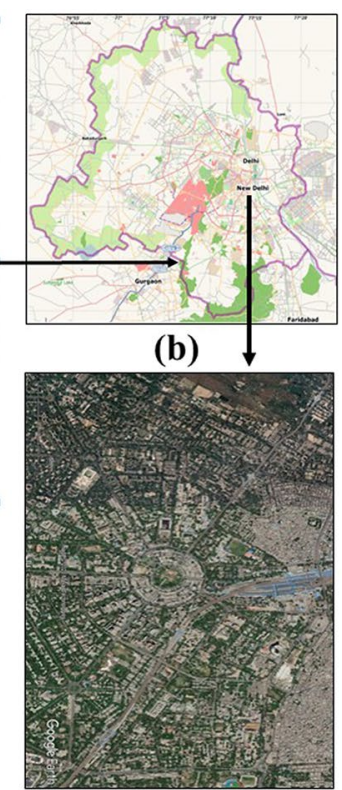

(c)

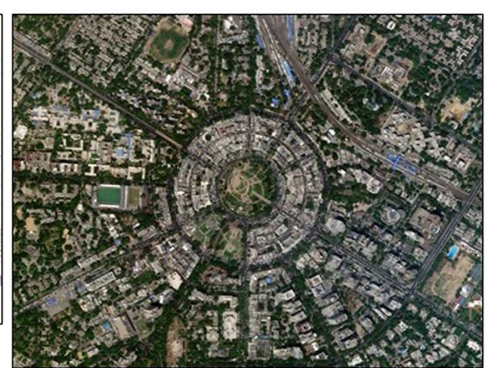

(d)

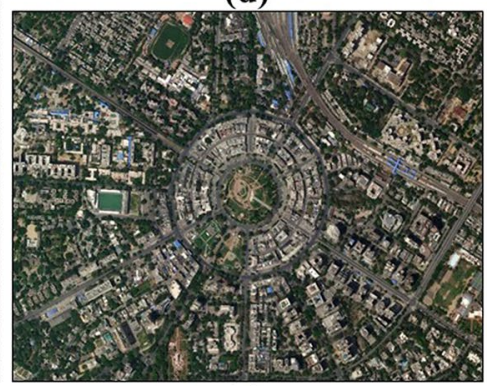

(e)

Fig. 11 Earthly location of the Connaught Place, New Delhi (Study area) 
Space Agency (Palermo 2018)," etc., that are actively working in the field of Earth exploration and remote sensing. Some prominent private players in space exploration include "SpaceX (Musk 2002)," "Boeing (Calhoun 1916)," "Sierra Nevada Corporation (Corporation 1963)," "Orbital (Thompson 1982)," etc. Skysat is a commercial microsatellite of Skybox imaging. This Earth observational satellite was developed to collect high-resolution multispectral and panchromatic images of the Earth's surface. This satellite universe consists of 21 satellites dedicated to Earth imaging. A private firm, "Planet own these satellites." First Skysat-1 was launched on 21 November 2013 (Marshall et al. 2010). Last year on 18 August 2020, Skysat 19-21 were launched (Marshall et al. 2010). The orbital type of Skysat 1-15 is sun-synchronous, whereas Skysat 16-21 is non-sun-synchronous. Skysat 1-2 has an orbital altitude of $600 \mathrm{~km}$, Skysat 3-15 has an orbital altitude of $500 \mathrm{~km}$, and Skysat 16-18 has an orbital length of $400 \mathrm{~km}$. The sensor installed on these satellite systems operates at spectral bandwidth of blue $450-515 \mathrm{~nm}$, green $515-595 \mathrm{~nm}$, red 605-695 nm, NIR 740-900 nm, and PAN 450-900 nm (Marshall et al. 2010).

In this research work, two panchromatic images of the pre-COVID and post-COVID (during lockdown) are obtained for the Skysat image database under the "research and training program" (Marshall et al. 2010). The preCOVID image and post-COVID image of the Connaught Place, New Delhi, investigated in this research work are represented in Fig. 11d, e. The study area's pre-COVID and post-COVID images are obtained on 30 April 2019 and 14 April 2020, respectively (Marshall et al. 2010). Connaught place's study area is popularly known as "Rajiv Chowk" (Hazarika et al. 2015). It is the leading financial and commercial center of the National capital of India. The study area is located at the heart of the National capital. It is having a latitude and longitude position of $28^{\circ} 37^{\prime} 58^{\prime}$ $\mathrm{N}$ and $77^{\circ} 13^{\prime} 11^{\prime} \mathrm{E}$ (Hazarika et al. 2015). The area of the Connaught place is $2.36 \mathrm{~km}^{2}$. The rapid urbanization in Connaught place has increased energy consumption and
Fig. 12 Pre-COVID image of the study area a RED Band, $\mathbf{b}$ GREEN Band, $\mathbf{c}$ BLUE Band, and $\mathbf{d}$ Stacked (RGB)
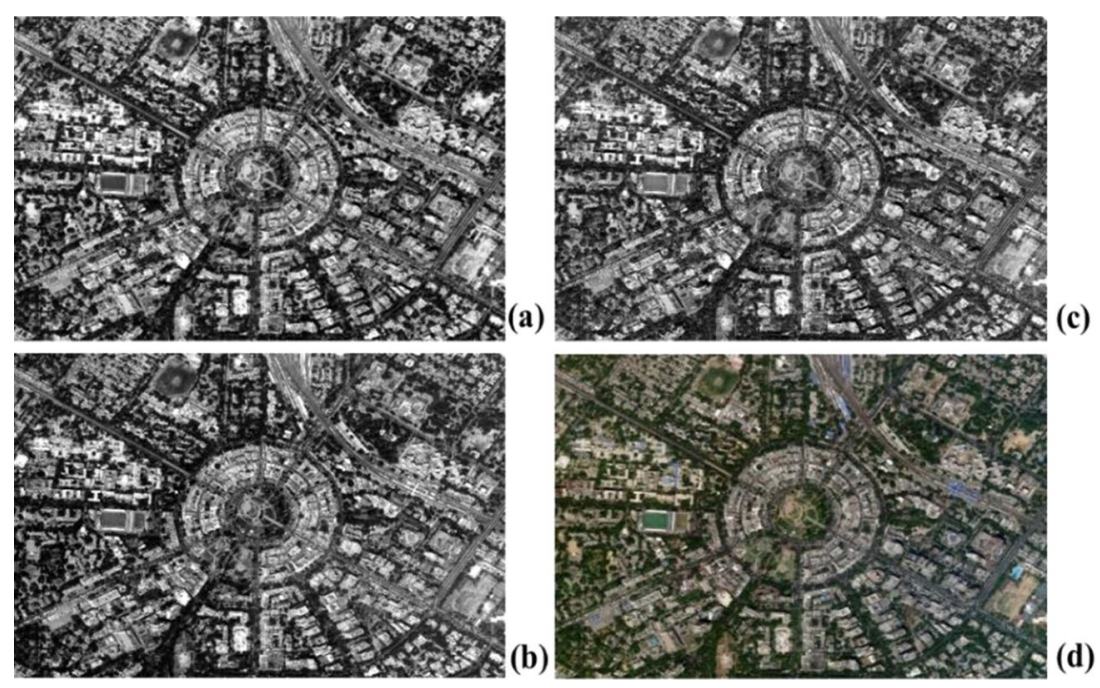

(d)
Fig. 13 Pre-COVID image. a Gray level representation. b Histogram signature plot

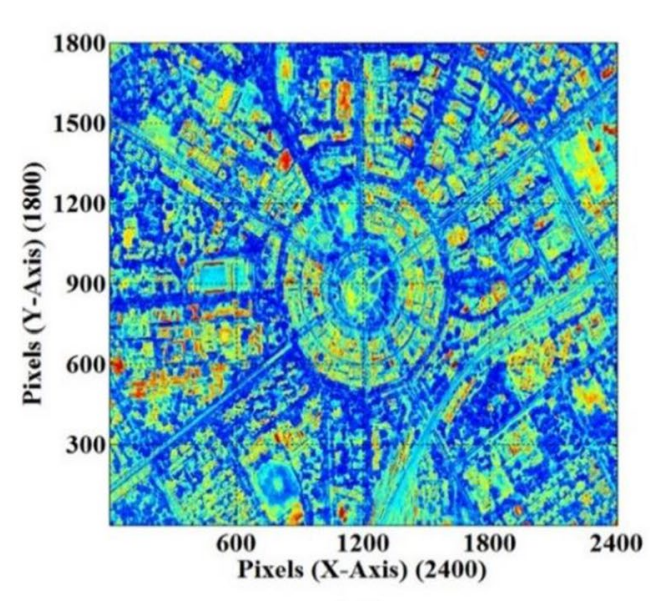

(a)

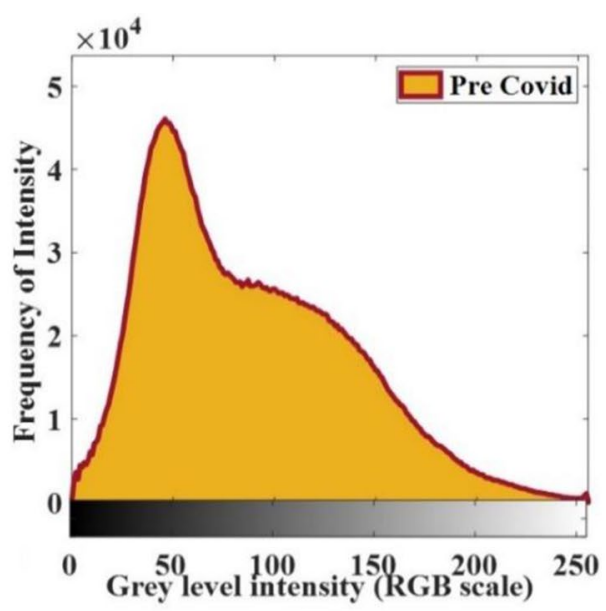

(b) 
Table 3 Quantification of the GLCM features for pre-COVID image

\begin{tabular}{|c|c|c|c|c|c|c|c|c|c|c|c|}
\hline \multirow{2}{*}{$\begin{array}{l}\text { Texture } \\
\text { GLCM features }\end{array}$} & \multirow{2}{*}{$\begin{array}{l}\text { Angle } \\
\text { Angular } \\
\text { orientation } \\
\left(^{\circ}\right)\end{array}$} & \multicolumn{8}{|c|}{ Variation in the distance (dis) } & \multicolumn{2}{|l|}{ Features } \\
\hline & & $\operatorname{dis}=1$ & $\operatorname{dis}=2$ & $\operatorname{dis}=3$ & $\operatorname{dis}=4$ & dis $=5$ & dis $=6$ & $\operatorname{dis}=7$ & $\operatorname{dis}=8$ & Sum features & Average features \\
\hline Contrast & 0 & 0.4527 & 0.8610 & 1.1757 & 1.4287 & 1.6420 & 1.8276 & 1.9975 & 2.1562 & 11.5414 & 1.4426 \\
\hline Correlation & 45 & 0.9042 & 0.8177 & 0.7511 & 0.6975 & 0.6524 & 0.6131 & 0.5772 & 0.5436 & 5.5568 & 0.6946 \\
\hline ASM & 90 & 0.1028 & 0.0806 & 0.0715 & 0.0655 & 0.0611 & 0.0578 & 0.0552 & 0.0530 & 0.5475 & 0.0684 \\
\hline IDM & 135 & 0.8252 & 0.7537 & 0.7171 & 0.6907 & 0.6699 & 0.6532 & 0.639 & 0.6269 & 5.5757 & 0.6969 \\
\hline Contrast & 0 & 0.7352 & 1.2436 & 1.6188 & 1.902 & 2.1416 & 2.3552 & 2.3569 & 2.7146 & 15.0679 & 1.8834 \\
\hline Correlation & 45 & 0.8443 & 0.7367 & 0.6573 & 0.5973 & 0.5466 & 0.5014 & 0.4608 & 0.4254 & 4.7698 & 0.5962 \\
\hline ASM & 90 & 0.0856 & 0.0708 & 0.0627 & 0.0577 & 0.0542 & 0.0515 & 0.0494 & 0.0478 & 0.4797 & 0.0599 \\
\hline IDM & 135 & 0.7724 & 0.7136 & 0.6763 & 0.6514 & 0.6324 & 0.6166 & 0.6033 & 0.5924 & 5.2584 & 0.6573 \\
\hline Contrast & 0 & 0.5065 & 0.9858 & 1.3112 & 1.5822 & 1.8095 & 1.9985 & 2.1675 & 2.3210 & 12.6822 & 1.5852 \\
\hline Correlation & 45 & 0.8928 & 0.7913 & 0.7224 & 0.665 & 0.6168 & 0.5768 & 0.5410 & 0.5085 & 5.3146 & 0.6643 \\
\hline ASM & 90 & 0.0976 & 0.0769 & 0.0689 & 0.0632 & 0.0592 & 0.0568 & 0.0538 & 0.0519 & 0.5283 & 0.0660 \\
\hline IDM & 135 & 0.8121 & 0.7401 & 0.7058 & 0.6789 & 0.6588 & 0.6431 & 0.6300 & 0.6190 & 5.4878 & 0.6859 \\
\hline Contrast & 0 & 0.6374 & 1.1029 & 1.4891 & 1.7935 & 2.0462 & 2.2635 & 2.4556 & 2.6266 & 14.4140 & 1.8018 \\
\hline Correlation & 45 & 0.8651 & 0.7665 & 0.6847 & 0.6203 & 0.5668 & 0.5208 & 0.4801 & 0.4440 & 4.9483 & 0.6185 \\
\hline ASM & 90 & 0.0889 & 0.073 & 0.0641 & 0.0586 & 0.0548 & 0.0519 & 0.0498 & 0.0481 & 0.4892 & 0.0611 \\
\hline IDM & 135 & 0.7845 & 0.7237 & 0.6839 & 0.6567 & 0.636 & 0.6195 & 0.6061 & 0.5948 & 5.3052 & 0.6631 \\
\hline
\end{tabular}

traffic density. Moreover the $\mathrm{PM}_{2.5}$ level of the Connaught place is highest in the National capital region (Shukla et al. 2020). Even the concentration of the $\mathrm{PM}_{2.5}$ level has even touched $999 \mu \mathrm{g} / \mathrm{m}^{3}$ during the worst time period (Mukherjee et al. 2020). The temperature of Connaught place raise to $45{ }^{\circ} \mathrm{C}$ during summers of April-June and falls to $8{ }^{\circ} \mathrm{C}$ during winters of December to January. Thus all these features significantly affect image classification and texture quantification.

\section{Experimental results}

\section{Change estimation through PBCD GLCM based technique}

In this investigation, three different band images of the study area are fused with the layer stacking technique available in ENVI 5.2. The layer stacking approach assists in generating a band-fused RGB image of the study area. The final image of the study area consists of all the essentials bands; thus GLCM method is applied to the study area. Figure 12a presents the RED band pre-COVID image of the study area, Fig. $12 \mathrm{~b}$ presents the GREEN band pre-COVID image of the study area and Fig. 12c presents the BLUE band pre-COVID image of the study area. Finally, all the bands are fused to create an RGB image of the study area presented in Fig. 12d.

The RGB image of the study area is converted to a gray level image to identify the changes in the study area represented in Fig. 13. The pixels count along the $X$-axis is 2400 pixels and along the $Y$-axis is 1800 pixels. Thus the total pixel count in the image is 4,320,000 pixels. Here, through the GLCM approach, one can identify the changes as a whole no separate object-based information is provided through GLCM. Figure 13a represents the grey level representation of the band fused pre-COVID image of the study area. Histogram signature plot of the band combined image represents the distribution of the frequency of the intensity value corresponding to the $4.32 \times 10^{6}$ image pixels illustrated in Fig. 13b. The visual texture features for the preCOVID image are quantified and presented in Table 3. Here the feature values are calculated corresponding to the total image pixels. Texture features are quantified corresponding to four orientation angles i.e. $0^{\circ}, 45^{\circ}, 90^{\circ}$ and $135^{\circ}$ and eight different distances $d=1, d=2, d=3, d=4, d=5, d=6$, $d=7$ and $d=8$. Later the texture features are averaged to make "GLCM direction independent."

The texture features contrast, correlation, ASM, and IDM are plotted to obtain their specific behavior. Concerning $0^{\circ}$ for eight different distances $d=1, d=2, d=3, d=4$, $d=5, d=6, d=7$, and $d=8$ it has been observed that "contrast" is sharply increasing with the orientation and distance. The correlation feature has presented a sharp decline in the quality. Energy features have indeed shown a reduction in the feature value, but the decline rate is prolonged. Finally, the feature IDM has also presented a sharp decline in the feature values similar to the decline rate of the correlation feature represented in Fig. 14a. 

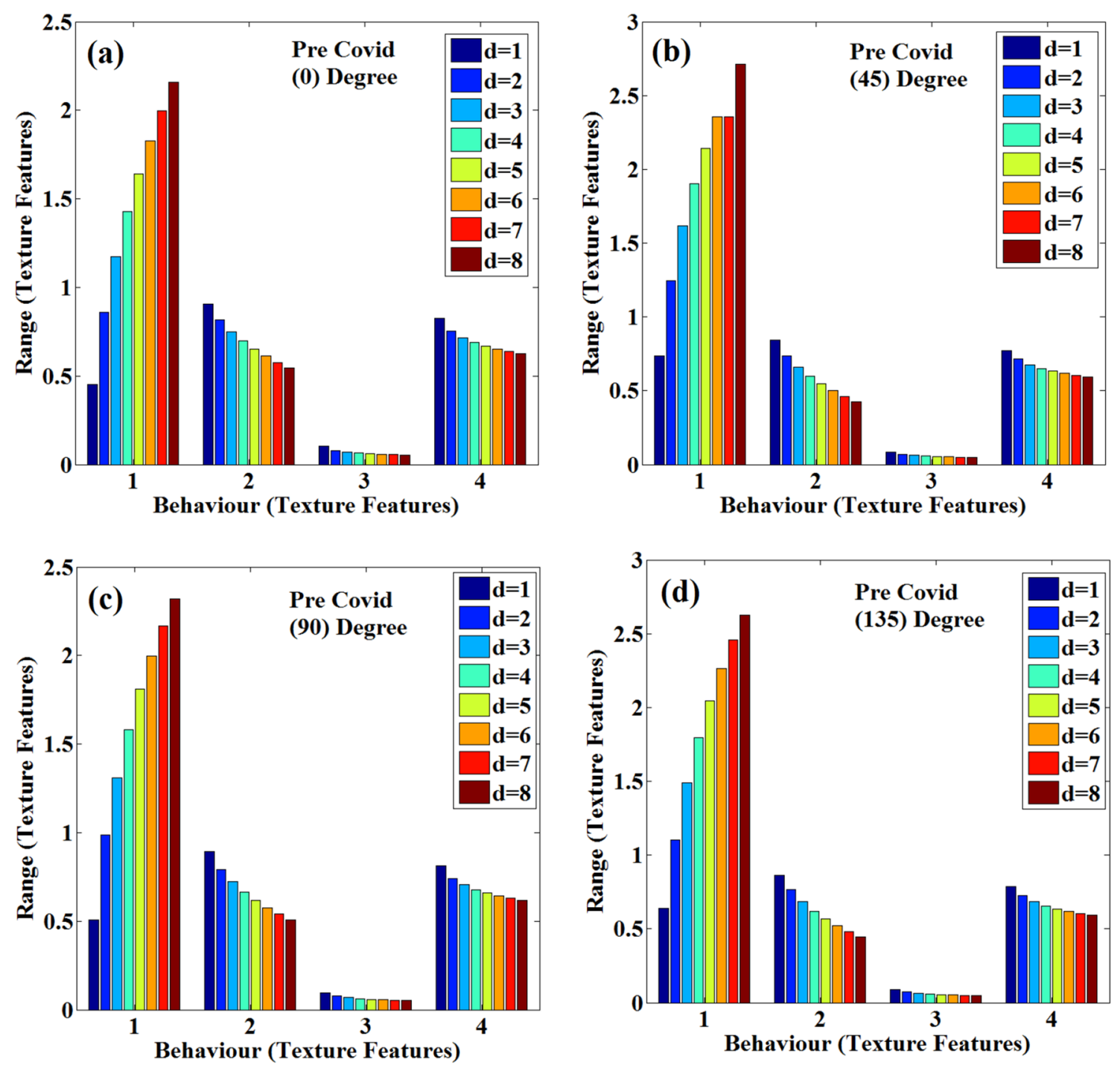

Fig. 14 Comparison of the texture features for pre-COVID image at different distances. a Pre-COVID $\left(0^{\circ}\right)$. b Pre-COVID $\left(45^{\circ}\right)$. c Pre-COVID $\left(90^{\circ}\right)$. d Pre-COVID $\left(135^{\circ}\right)$

Fig. 15 Post-COVID image of the study area. a RED Band, b GREEN Band, c BLUE Band, and $\mathbf{d}$ Stacked (RGB) band
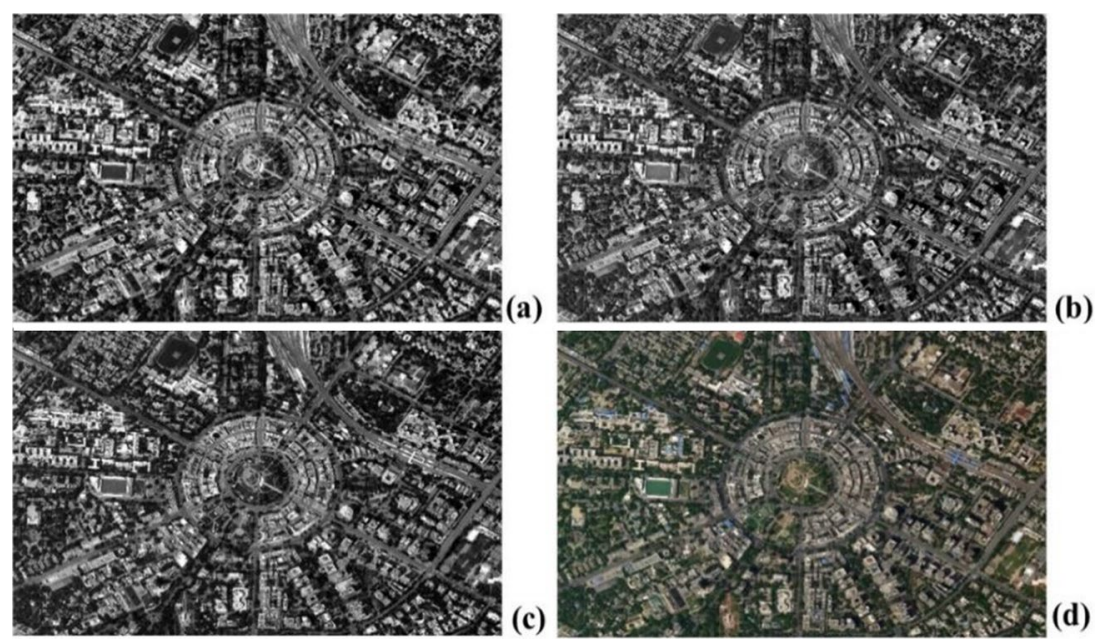

(c)

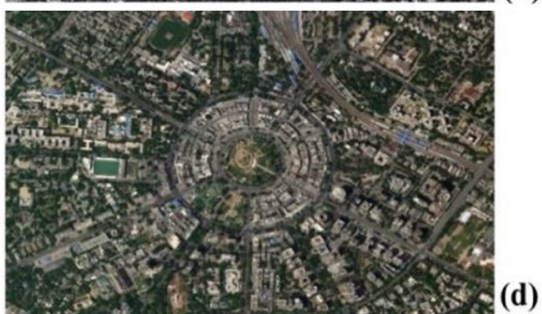


Fig. 16 Post-COVID image. a Gray level representation. b Histogram signature plot

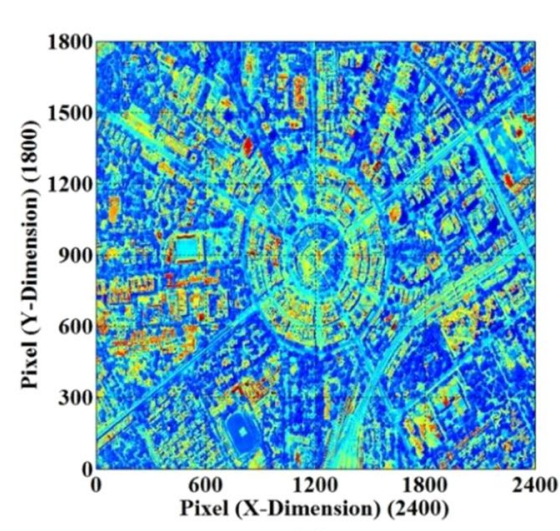

(a)

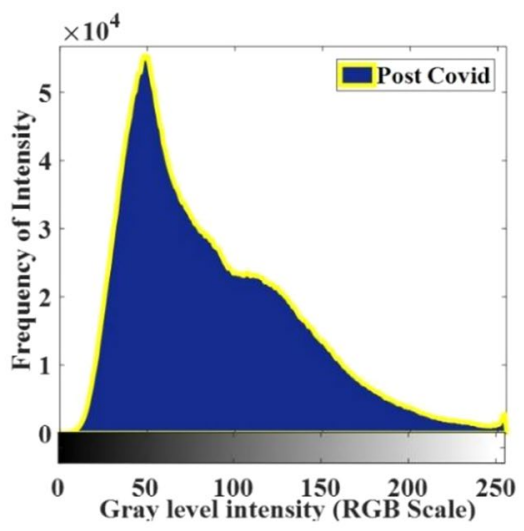

(b)

Table 4 Quantification of the GLCM feature for post-COVID image

\begin{tabular}{|c|c|c|c|c|c|c|c|c|c|c|c|}
\hline \multirow{2}{*}{$\begin{array}{l}\text { Texture } \\
\text { GLCM features }\end{array}$} & \multirow{2}{*}{$\begin{array}{l}\text { Angle } \\
\text { Angular } \\
\text { orientation } \\
\left({ }^{\circ}\right)\end{array}$} & \multicolumn{8}{|c|}{ Variation in the distance (dis) } & \multicolumn{2}{|l|}{ Features } \\
\hline & & $\operatorname{dis}=1$ & dis $=2$ & $\operatorname{dis}=3$ & $\operatorname{dis}=4$ & dis $=5$ & $\operatorname{dis}=6$ & $\operatorname{dis}=7$ & $\operatorname{dis}=8$ & Sum features & Average features \\
\hline Contrast & 0 & 0.4527 & 0.8610 & 1.1757 & 1.4287 & 1.6420 & 1.8276 & 1.9975 & 2.1562 & 11.5414 & 1.4426 \\
\hline Correlation & 45 & 0.9042 & 0.8177 & 0.7511 & 0.6975 & 0.6524 & 0.6131 & 0.5772 & 0.5436 & 5.5568 & 0.6946 \\
\hline ASM & 90 & 0.1028 & 0.0806 & 0.0715 & 0.0655 & 0.0611 & 0.0578 & 0.0552 & 0.0530 & 0.5475 & 0.0684 \\
\hline IDM & 135 & 0.8252 & 0.7537 & 0.7171 & 0.6907 & 0.6699 & 0.6532 & 0.6390 & 0.6269 & 5.5757 & 0.6969 \\
\hline Contrast & 0 & 0.7352 & 1.2436 & 1.6188 & 1.9020 & 2.1416 & 2.3552 & 2.3569 & 2.7146 & 15.0679 & 1.8834 \\
\hline Correlation & 45 & 0.8443 & 0.7367 & 0.6573 & 0.5973 & 0.5466 & 0.5014 & 0.4608 & 0.4254 & 4.7698 & 0.5962 \\
\hline ASM & 90 & 0.0856 & 0.0708 & 0.0627 & 0.0577 & 0.0542 & 0.0515 & 0.0494 & 0.0478 & 0.4797 & 0.0599 \\
\hline IDM & 135 & 0.7724 & 0.7136 & 0.6763 & 0.6514 & 0.6324 & 0.6166 & 0.6033 & 0.5924 & 5.2584 & 0.6573 \\
\hline Contrast & 0 & 0.5065 & 0.9858 & 1.3112 & 1.5822 & 1.8095 & 1.9985 & 2.1675 & 2.3210 & 12.6822 & 1.5852 \\
\hline Correlation & 45 & 0.8928 & 0.7913 & 0.7224 & 0.6650 & 0.6168 & 0.5768 & 0.5410 & 0.5085 & 5.3146 & 0.6643 \\
\hline ASM & 90 & 0.0976 & 0.0769 & 0.0689 & 0.0632 & 0.0592 & 0.0568 & 0.0538 & 0.0519 & 0.5283 & 0.0660 \\
\hline IDM & 135 & 0.8121 & 0.7401 & 0.7058 & 0.6789 & 0.6588 & 0.6431 & 0.6300 & 0.6190 & 5.4878 & 0.6859 \\
\hline Contrast & 0 & 0.6374 & 1.1029 & 1.4891 & 1.7935 & 2.0462 & 2.2635 & 2.4556 & 2.6266 & 14.4148 & 1.8018 \\
\hline Correlation & 45 & 0.8651 & 0.7665 & 0.6847 & 0.6203 & 0.5668 & 0.5208 & 0.4801 & 0.4440 & 4.9483 & 0.6185 \\
\hline ASM & 90 & 0.0889 & 0.0730 & 0.0641 & 0.0586 & 0.0548 & 0.0519 & 0.0498 & 0.0481 & 0.4892 & 0.0611 \\
\hline IDM & 135 & 0.7845 & 0.7237 & 0.6839 & 0.6567 & 0.6360 & 0.6195 & 0.6061 & 0.5948 & 5.3052 & 0.6631 \\
\hline
\end{tabular}

Similarly, the GLCM feature behavior corresponding to a $45^{\circ}$ is presented in Fig. $14 \mathrm{~b}$, corresponding to a $90^{\circ}$ is introduced in Fig. $14 \mathrm{c}$ and corresponding to a $135^{\circ}$ is illustrated in Fig. 14d. It has been observed that GLCM features have obtained the same pattern in the quantified feature values for different orientation angles. Thus from this analysis, new information about the GLCM is discovered that this approach can also be used for pattern recognition. GLCM is mainly considered an approach for texture classification, but GLCM has provided information about the image texture as a whole; not specific details on any classified object can be identified from the GLCM. Similarly, the post-COVID image of the study area is explored, and texture feature quantification and feature plotting are performed to obtain new information.

The post-COVID image analysis of the study area is presented in Fig. 15. Figure 15a represents the RED band image, Fig. 15b presents the GREEN band image, and Fig. $15 \mathrm{c}$ represents the BLUE band image of the study area. All the images are fused to obtain a multiband RGB image of the study area. Some general assumption about the postCOVID image is that low or no traffic on the streets will be observed. Due to the continuous lockdown situation, it is expected that the air quality will also be undoubtedly improved. Thus it will be interesting to observe the behavior of the texture features for the post-COVID image. The layer 

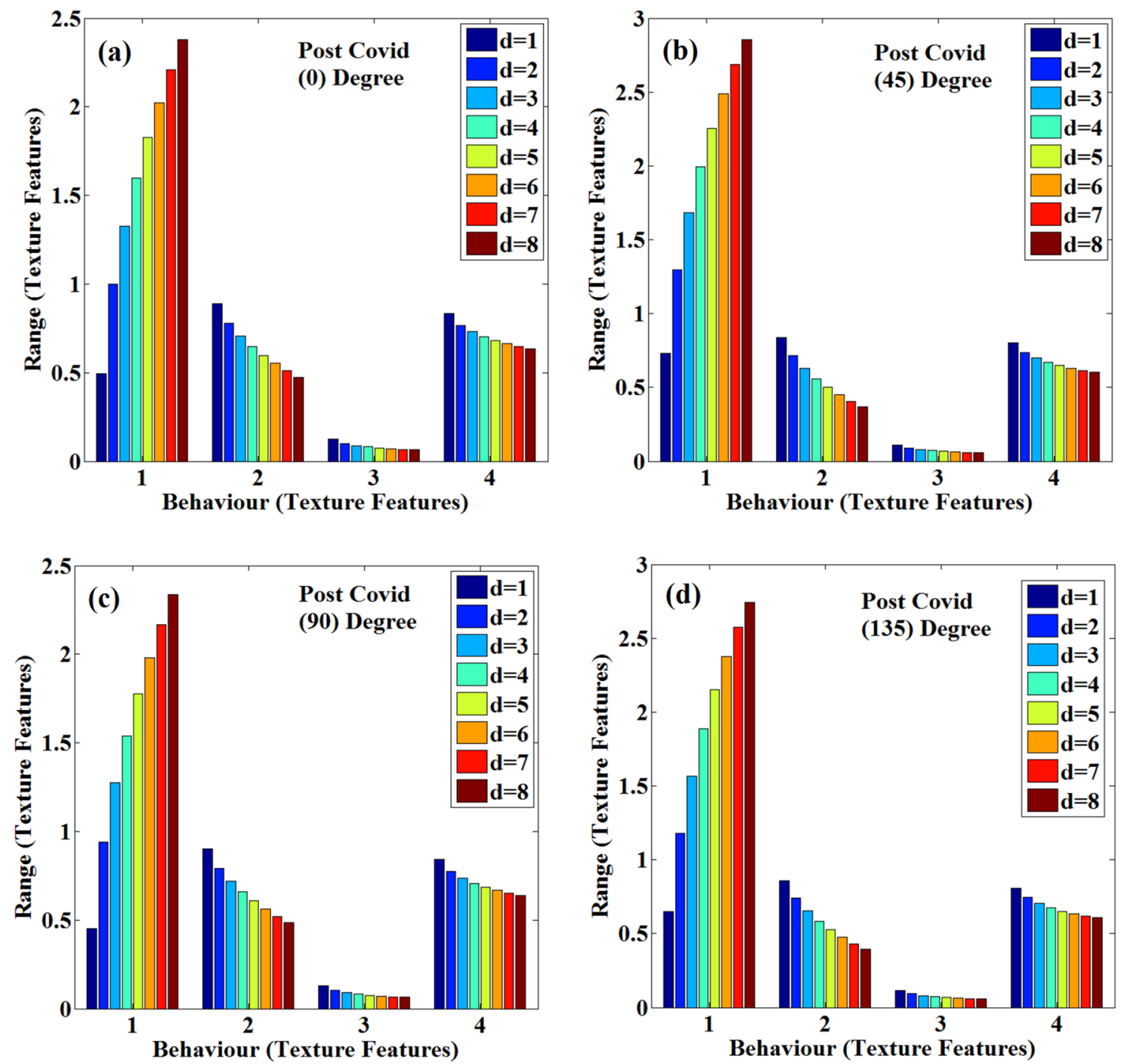

Fig. 17 Comparison of the texture features for post-COVID image at different distances. a Post-COVID $\left(0^{\circ}\right)$. b Post-COVID $\left(45^{\circ}\right)$. c PostCOVID $\left(90^{\circ}\right)$. d Post-COVID $\left(135^{\circ}\right)$

stacking technique available with ENVI 5.2 is adopted to perform band fusion and develop an RGB image of the study area. Figure $15 \mathrm{~d}$ represents the band fused post-COVID image of the study area.

The grey level representation of the post-COVID study area is presented in Fig. 16. Figure 16a represents the postCOVID image of the study area, which visually looks similar to the pre-COVID image. No significant change is visually identified in the study area. The quantification of the texture features can obtain a difference in the study area, and the modifications are developed in the histogram signature plot of the study area. The histogram signature plot in Fig. 16b visually represents the changing pattern of the surface of the study area. The quantification of the texture features for the post-COVID image is presented in Table 4.
The texture features for the post-COVID image seem to be identical with the texture features of the pre-COVID image shown in Fig. 17. The behavior of the texture feature "contrast" has shown an increasing pattern for $0^{\circ}, 45^{\circ}, 90^{\circ}$ and $135^{\circ}$. Texture features correlation has shown decreasing pattern for all four degrees. The decrease of these texture features is high compared to the remaining texture features for all four orientations. Texture feature ASM has shown a minimum decrease rate. Finally, texture feature IDM has also shown decreasing pattern for all four directions. Here it has been observed that when GLCM is used to compare two multi-band images. It may be possible that the change developed in the two images can be visually identified. Histogram signature plotting is a method to determine the occurrence of changes. The most important procedure 

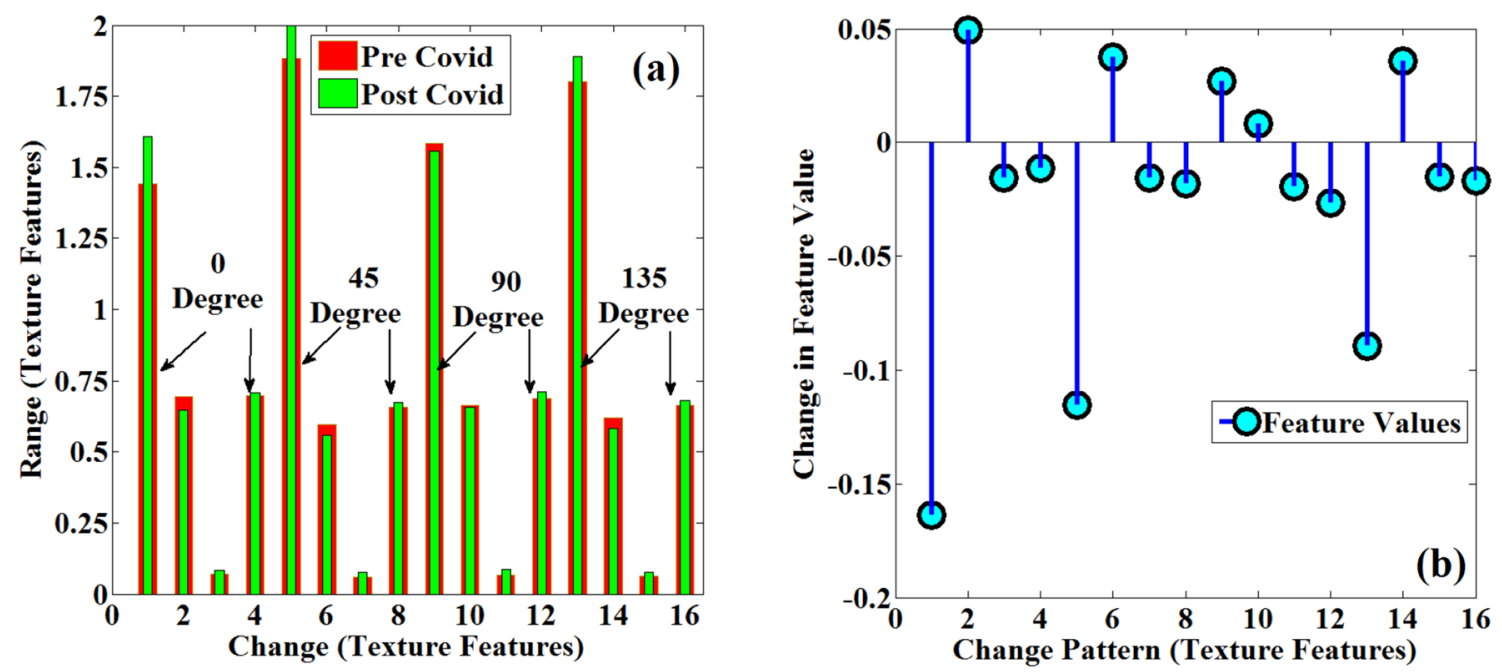

Fig. 18 a Change in the texture features corresponding to pre-COVID and post-COVID image. b Change pattern analysis of the feature values

\begin{tabular}{|c|c|c|c|c|c|}
\hline \multirow{2}{*}{$\begin{array}{c}\text { Classification } \\
\text { Classes }\end{array}$} & \multicolumn{5}{|c|}{ Reference value } \\
\hline & Water & Vegetation & Urban & Soil & Rows Total \\
\hline Water & 35 & 9 & 9 & 11 & 64 \\
\hline Vegetation & 8 & 44 & 7 & 9 & 68 \\
\hline Urban & 9 & 15 & 152 & 14 & 190 \\
\hline Soil & 11 & 8 & 7 & 59 & 85 \\
\hline Column Total & 63 & 76 & 175 & 93 & 407 \\
\hline
\end{tabular}

Fig. 19 Test data for the image classification

to detect the developed changes is the comparison of the average texture features. As discussed earlier, an average of the GLCM features is done to make the GLCM direction independent. Figure 18a compares the texture features for the pre-COVID and post-COVID images. Here it can be seen that "contrast" has obtained the highest peak value. Contrast is highest for $0^{\circ}, 45^{\circ}, 135^{\circ}$ with corresponds to post-COVID image, for $90^{\circ}$ contrast for pre-COVID image is high. Correlation has obtained a high-value corresponding to all orientations for the pre-COVID image. ASM has received a high value for all directions corresponding to the post-COVID image. Finally, IDM received a high value for all orientations corresponding to the post-COVID image. Thus, texture features have obtained high feature values for the post-COVID image for most cases. Figure 18b represents the change in the texture features along the positive or negative end. Five times, the difference of texture features for pre-COVID and post-COVID images have produced positive values, and on eleven occasions, texture features have negative values. This also suggests that post-COVID image texture features have obtained higher values than the pre-COVID image.

\section{Change analysis through object-based image classification techniques}

While performing image classification through object-based image classification techniques. A particular area is classified into several objects. These objects are geographical areas of the study area like water, land, soil, tree, etc. Some of the prominent terms associated with the object-based image classification through which the image classification is performed are UA, PA, CE, OE, OA and $K_{p}$. The procedure to calculate these image classification features can be understood by the example of a confusion matrix represented in Fig. 19. Here a confusion matrix with arbitrary image classification is assumed. This random image is classified into four different classes as water, vegetation, urban, and soil.

Now different parameters corresponding to the image classification are computed as follows.

Commission error $(C E)$ is generated when the pixels of one class get wrongly introduced in the class under observation. CE concerning the classified categories can be expressed by Eqs. (26-29).

$\mathrm{CE}($ water $)=(9+9+11) / 64=29 / 64=0.4531=45.31 \%$

$\mathrm{CE}($ vegetation $)=(8+7+9) / 68=24 / 68=0.3529=35.29 \%$

$\mathrm{CE}($ urban $)=(9+5+14) / 190=38 / 190=0.20=20 \%$

$\mathrm{CE}($ soil $)=(11+8+7) / 85=26 / 85=0.3058=30.58 \%$

Omission error $(O E)$ refers to the classified pixels that are accidentally omitted from the investigation classes. OE concerning the classified classes is expressed by Eqs. (30)-(33). 
$\mathrm{OE}($ water $)=(8+9+11) / 63=28 / 63=0.4444=44.44 \%$

$\mathrm{OE}($ vegetation $)=(9+15+8) / 76=32 / 76=0.4210=42.10 \%$

$\mathrm{OE}($ urban $)=(9+7+7) / 175=23 / 175=0.1314=13.14 \%$

OE $($ soil $)=(11+9+14) / 93=34 / 93=0.3655=36.55 \%$

User accuracy (UA) is defined as the "accuracy" from the perspective of the "map user," not from the perspective of the "map maker." UA concerning the classified classes are expressed by Eq. (34)-(38).

User accuracy $=100 \%-$ commission error

$\mathrm{UA}($ water $)=100-\mathrm{CE}($ water $)=100-45.31=54.69 \%$

$\mathrm{UA}($ vegetation $)=100-\mathrm{CE}($ vegetation $)=100-35.29=64.71 \%$

$\mathrm{UA}($ urban $)=100-\mathrm{CE}($ urban $)=100-20=80.00 \%$

$\mathrm{UA}($ soil $)=100-\mathrm{CE}($ soil $)=100-30.58=69.42 \%$

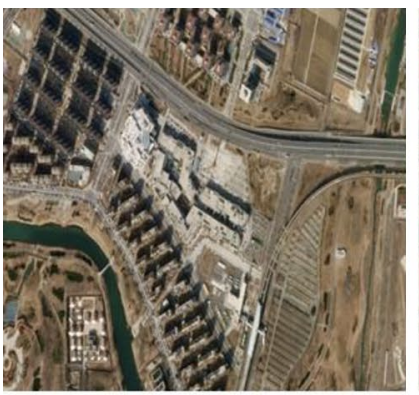

(a)

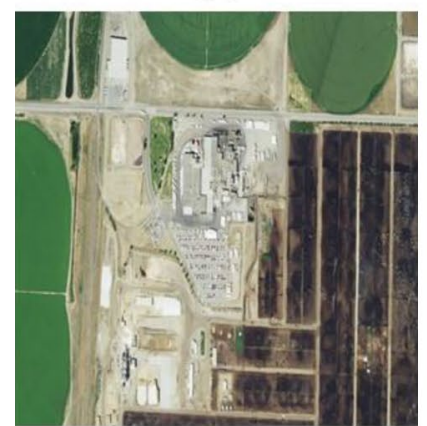

(e)

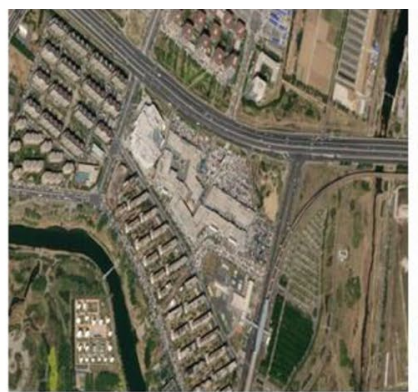

(b)

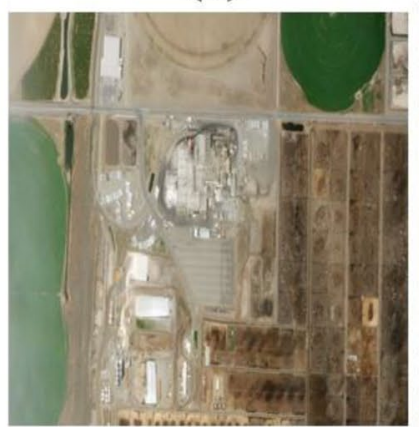

(f)

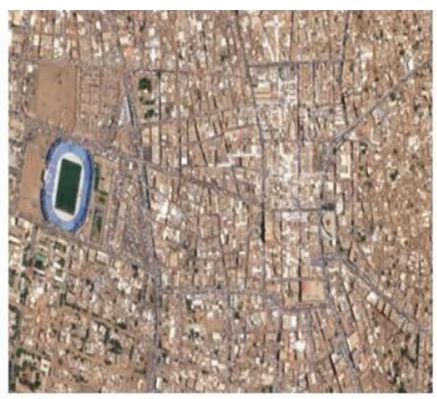

(c)

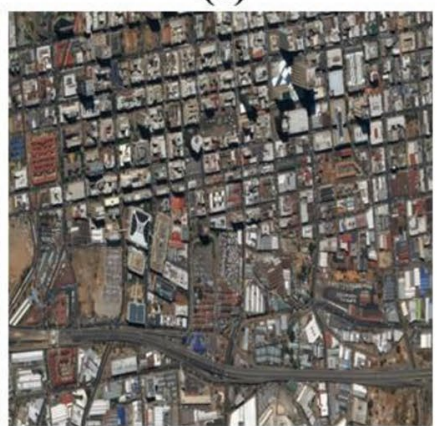

(g)

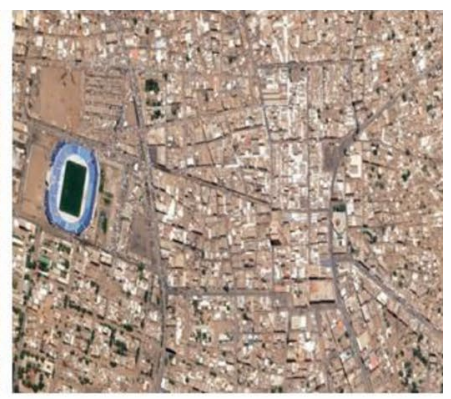

(d)

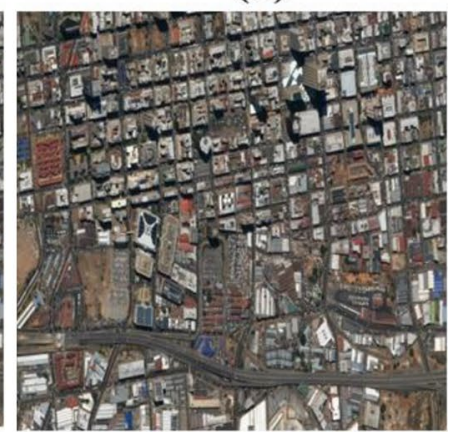

(h)
Fig. 20 Pre and post-COVID Skysat images of the various location, pre-COVID. a China, Beijing (12 April 2020), c Sudan Omdurman, (23 April 2020), e Tyson foods, Washington, USA (30 April 2020), g South Africa, Johannesburg (27 September 2019); post-COVID.
Producer accuracy $(P A)$ is defined as the "accuracy" the perspective of the "map maker," not from the "map user." PA concerning the classified Producer accuracy $=100 \%-$ ommission error

$\mathrm{PA}($ vegetation $)=100-\mathrm{OE}($ vegetation $)=100-42.10=57.90 \%$

$\mathrm{PA}($ urban $)=100-\mathrm{OE}($ urban $)=100-13.14=86.86 \%$

Overall accuracy $(O A)$ can be understood as a ratio between the "correctly classified pixels" to the "total number of pixels" present in the image. It is expressed by Eq. (44).

Overall accuracy $(\mathrm{OA})=\frac{\text { Correctly classified pixels }}{\text { Total numbers of the image pixels }}$

OA for the confusion matrix presented in Fig. 19 is calculated as $\mathrm{OA}=\frac{35+44+152+59}{407}=\frac{290}{407}=0.7125=71.25 \%$.

b China, Beijing (12 April 2020), d Sudan, Omdurman (23 April 2020), f Tyson foods, Washington, USA (30 April 2020), h South Africa, Johannesburg (27 September 2019) 
Table 5 Pixel counts for training and accuracy assessment of study area

\begin{tabular}{llllll}
\hline S. no. & Class & Training pixels & $\begin{array}{l}\text { Accuracy assess- } \\
\text { ment pixels }\end{array}$ & Training pixels & $\begin{array}{l}\text { Accuracy } \\
\text { assessment } \\
\text { pixels }\end{array}$ \\
& & Pre-COVID image & & Post-COVID image \\
\hline 1 & Building & 1471 & 1611 & 1458 & 1544 \\
2 & Tree & 1090 & 1469 & 1107 & 1484 \\
3 & Road & 1540 & 1095 & 1678 & 1578 \\
4 & Grassland & 1748 & 1723 & 1478 & 1536 \\
5 & Metro & 1898 & 2183 & 1788 & 1849 \\
6 & Car & 1026 & 1024 & 1395 & 1748 \\
\hline
\end{tabular}

Thus the OA classification accuracy for the presented confusion matrix is $71.25 \%$.

Finally, the $K_{p}$ is calculated to obtain classification accuracy. It is the measure of how well the classification of the study area dataset is performed. The range of $K_{p}$ is $[-1,1]$. Mathematically it is expressed by Eq. (45).
If $K_{p}$ is lying close to -1 , then it is assumed to be a worse image classification.

If $K_{p}$ is lying close to 0 , then it is random image classification.

If $K_{p}$ is lying close to +1 , then the classification is assumed to be significantly realistic and close to accurate.

Kappa coefficient $=\frac{\operatorname{probablity}(O)-\operatorname{probablity}(e)}{1-\operatorname{probablity}(e)}=1-\frac{1-\operatorname{probablity}(O)}{1-\operatorname{probablity}(e)}$

where probablity $(O)$ is considered as the "observed agreement" among various classification raters. This is assumed to be identical with accuracy. Probablity $(e)$ is considered as a "theoretical probability of the chance agreement." While performing the image classification using the $K_{p}$ following assumptions are kept into consideration.
In early 2020, coronavirus was assumed to be spread in the entire world. In the USA, during early January of 2020, coronavirus was considered to have limited spread (Jorden et al. 2020). In China, cases related to the novel coronavirus began to report from late 2019 (Xu et al. 2020). Issues related to coronavirus were highlighted in early 2020, but

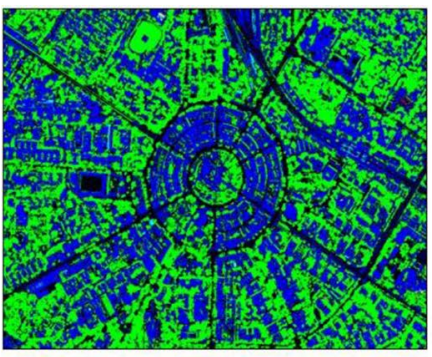

(a)

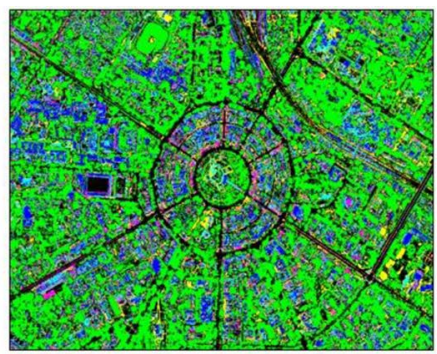

(b)
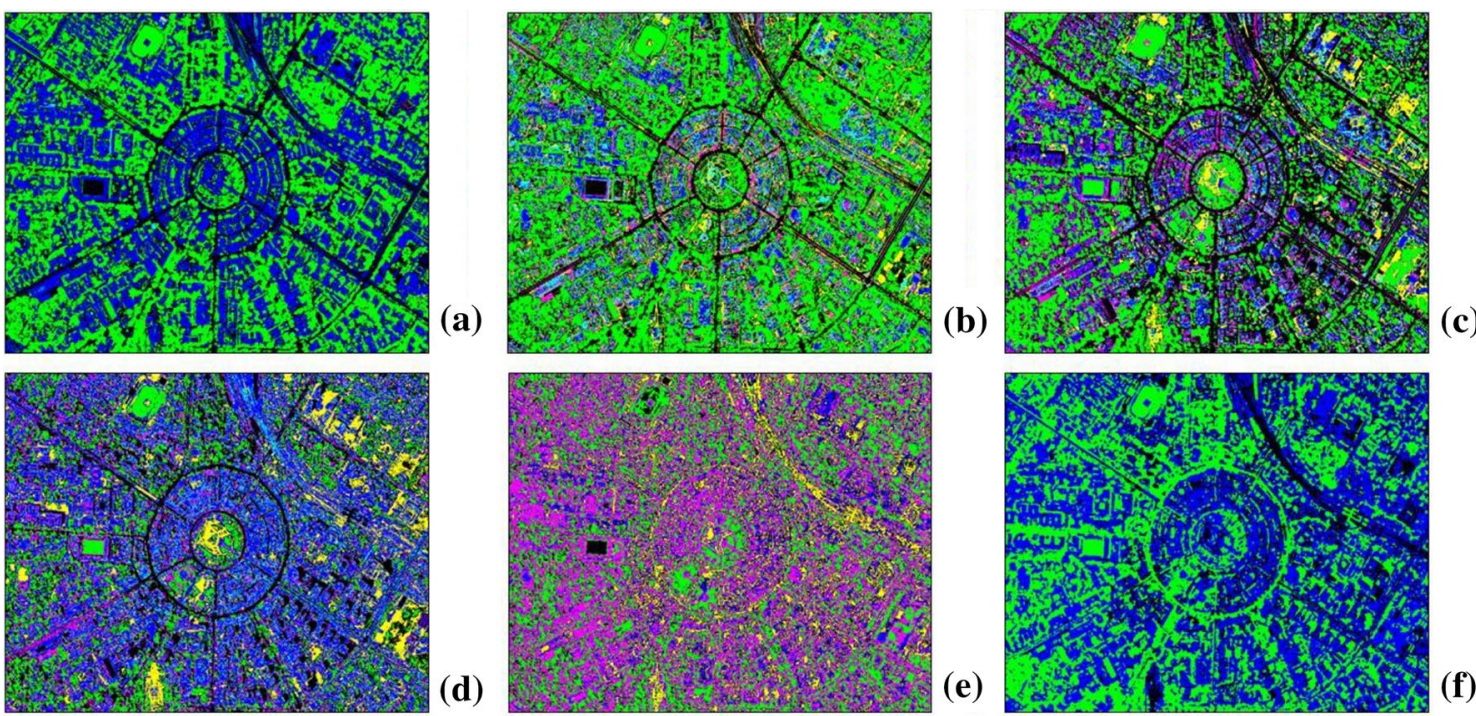

Building (d)

Roads
Grasslands

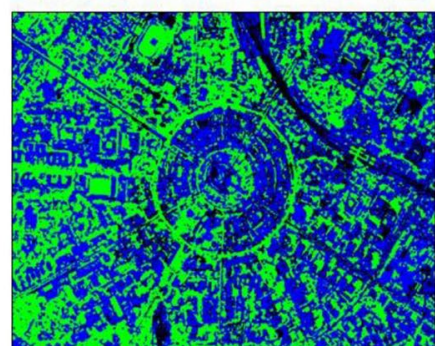

(f)
Metro

Fig. 21 Pre-COVID image classification. a "Parallelepiped classification", b "Minimum distance classification", c "Maximum likelihood classification", d "Spectral angle mapper", e "Spectral Information Divergence", f "Support Vector Machine" 
Table 6 Classification features for the pre-COVID classified image

\begin{tabular}{|c|c|c|c|c|c|c|c|}
\hline \multirow[t]{2}{*}{ Scheme } & \multirow[t]{2}{*}{ Classification class } & \multicolumn{4}{|c|}{ Classification features } & \multirow[t]{2}{*}{ Overall accuracy } & \multirow[t]{2}{*}{ Kappa coefficient } \\
\hline & & UA & PA & $\mathrm{OE}$ & $\mathrm{CE}$ & & \\
\hline \multirow[t]{6}{*}{ Parallelepiped classification } & Building & 31.50 & 92.55 & 7.45 & 68.50 & \multirow[t]{6}{*}{$(3841 / 6428)=59.7542 \%$} & \multirow[t]{6}{*}{0.3068} \\
\hline & Tree & 63.98 & 100.00 & 0.00 & 36.02 & & \\
\hline & Road & 34.74 & 51.14 & 48.86 & 65.26 & & \\
\hline & Grassland & 0.00 & 0.00 & 100.00 & 100.00 & & \\
\hline & Metro & 73.04 & 14.52 & 85.48 & 26.96 & & \\
\hline & Car & 100.00 & 0.39 & 99.61 & 0.00 & & \\
\hline \multirow[t]{6}{*}{ Minimum distance classification } & Building & 76.97 & 75.04 & 24.96 & 23.03 & \multirow[t]{6}{*}{$(4821 / 6428)=75.0000 \%$} & \multirow[t]{6}{*}{0.6853} \\
\hline & Tree & 99.44 & 96.32 & 3.68 & 0.56 & & \\
\hline & Road & 53.32 & 99.54 & 0.46 & 46.68 & & \\
\hline & Grassland & 79.59 & 90.20 & 9.80 & 20.41 & & \\
\hline & Metro & 45.83 & 1.97 & 98.03 & 54.17 & & \\
\hline & Car & 48.54 & 24.11 & 75.89 & 51.46 & & \\
\hline \multirow[t]{6}{*}{ Maximum likelihood classification } & Building & 75.41 & 82.65 & 17.35 & 24.59 & \multirow[t]{6}{*}{$(4149 / 6428)=64.5457 \%$} & \multirow[t]{6}{*}{0.7885} \\
\hline & Tree & 100.00 & 100.00 & 0.00 & 0.00 & & \\
\hline & Road & 88.97 & 93.81 & 6.19 & 11.03 & & \\
\hline & Grassland & 91.59 & 75.55 & 24.45 & 8.41 & & \\
\hline & Metro & 51.77 & 45.61 & 54.39 & 48.23 & & \\
\hline & Car & 53.48 & 30.47 & 69.53 & 46.52 & & \\
\hline \multirow[t]{6}{*}{ Spectral angle mapper } & Building & 42.02 & 58.85 & 41.15 & 57.98 & \multirow[t]{6}{*}{$(3508 / 6428)=54.5737 \%$} & \multirow[t]{6}{*}{0.2791} \\
\hline & Tree & 100.00 & 19.61 & 80.39 & 0.00 & & \\
\hline & Road & 54.29 & 17.35 & 82.65 & 45.71 & & \\
\hline & Grassland & 71.96 & 96.81 & 3.19 & 28.04 & & \\
\hline & Metro & 0.00 & 0.00 & 100.00 & 100.00 & & \\
\hline & Car & 25.17 & 40.43 & 59.57 & 74.83 & & \\
\hline \multirow[t]{6}{*}{ Spectral information divergence } & Building & 28.21 & 33.46 & 66.54 & 76.79 & \multirow[t]{6}{*}{$(1494 / 6428)=23.2420 \%$} & \multirow[t]{6}{*}{0.0154} \\
\hline & Tree & 81.36 & 12.19 & 87.81 & 18.64 & & \\
\hline & Road & 35.09 & 21.92 & 78.08 & 64.91 & & \\
\hline & Grassland & 11.28 & 8.94 & 91.06 & 88.72 & & \\
\hline & Metro & 16.51 & 10.58 & 89.42 & 83.49 & & \\
\hline & Car & 5.22 & 14.75 & 85.25 & 94.78 & & \\
\hline \multirow[t]{6}{*}{ Support vector machine } & Building & 66.92 & 65.05 & 34.95 & 33.08 & \multirow[t]{6}{*}{$(1332 / 6428)=20.7218 \%$} & \multirow[t]{6}{*}{0.5131} \\
\hline & Tree & 98.94 & 89.38 & 10.62 & 1.06 & & \\
\hline & Road & 37.31 & 95.98 & 4.02 & 62.69 & & \\
\hline & Grassland & 98.46 & 96.40 & 3.60 & 1.54 & & \\
\hline & Metro & 0.00 & 0.00 & 100.00 & 100.00 & & \\
\hline & Car & 16.23 & 25.29 & 74.71 & 83.77 & & \\
\hline
\end{tabular}

the government of South Africa imposed a lockdown by March 2020 (Atangana and Araz 2020). Figure 20 presents the geographical locations of some prominent places before and during the lockdown. Here the pre lockdown images represent normal day-to-day activities of the people. A sudden stop in everyday activities is reported during the lockdown, i.e., no people on the road, empty roads, no industrial activities, etc.

The pre and post-study area images are classified using six different image classification techniques, i.e., PC, MDC, MLC, SAM, SID, and SVM. The image classification is performed based on six other classes, i.e., buildings, trees, roads, grasslands, metro, and cars. Two different sets of ROIs are created for classifying the images. ROI set 1 contain image pixels for training the data and ROI set 2 image pixels for the accuracy assessment. Table 5 presents details of the pixel's count collected for training and accuracy assessment of the image data. Pre-COVID classification results of the study area are shown in Fig. 21.

The image classification features for different classification schemes are tabulated in Table 6. 


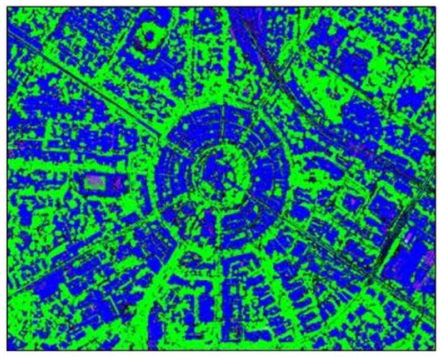

(a)

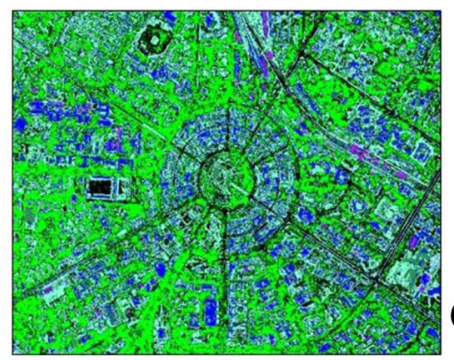

(b)
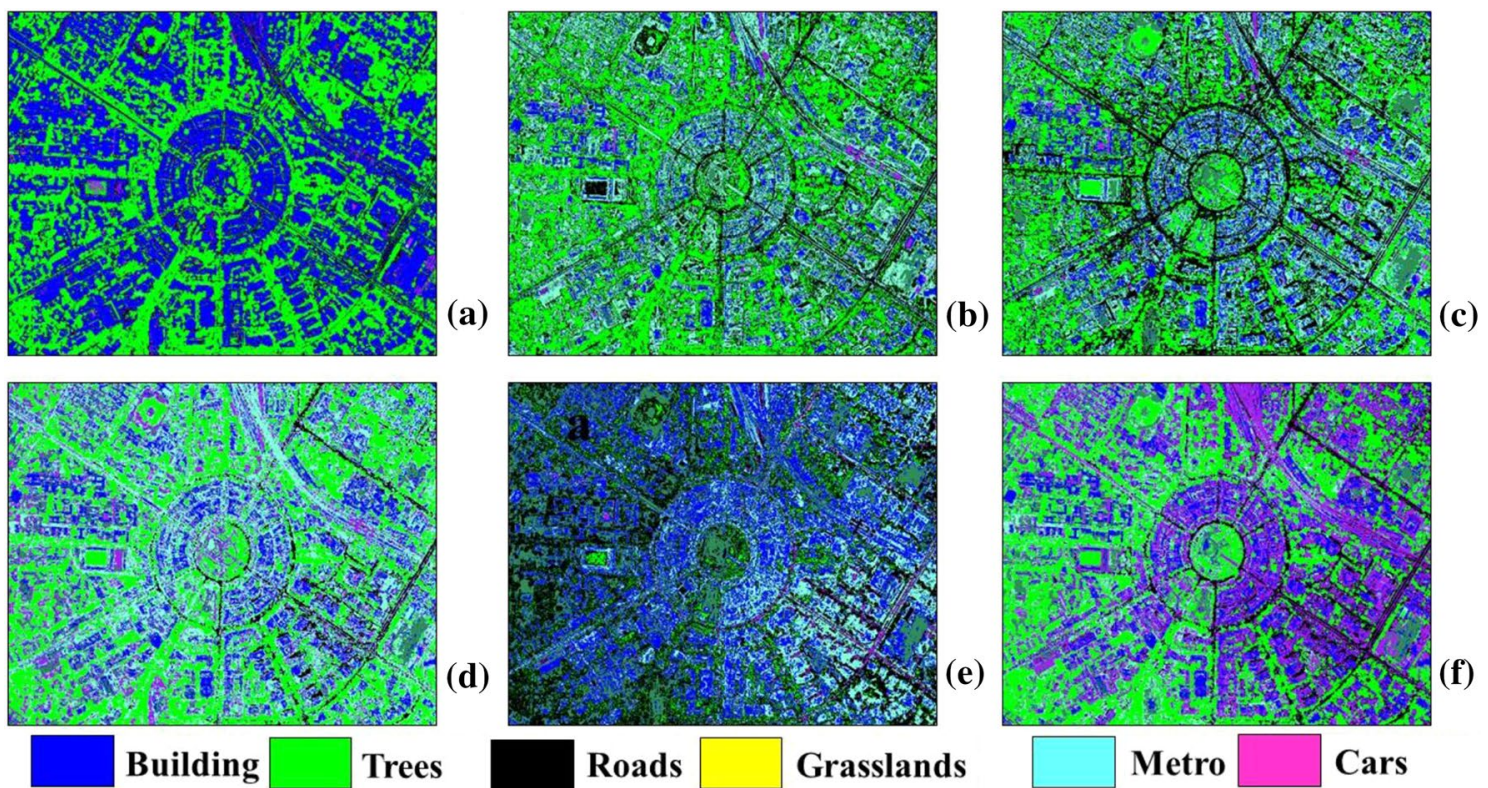

(d)

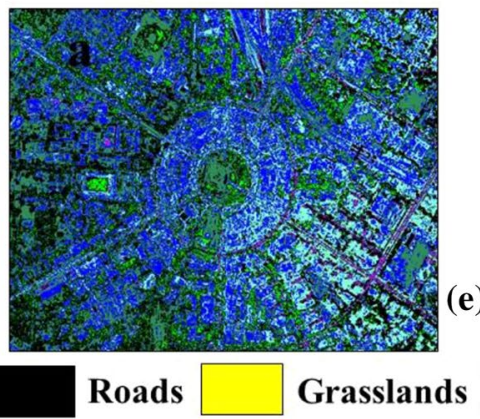

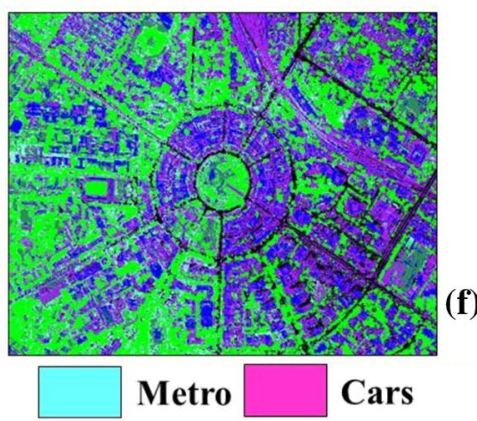

Fig. 22 Post-COVID image classification. a "Parallelepiped classification", b "Minimum distance classification", c "Maximum likelihood classification", d "Spectral angle mapper", e "Spectral Information Divergence", f "Support Vector Machine"

The image classification of the post-COVID study area is performed and presented in Fig. 22. The image classification features for different classification schemes obtained for the post-COVID image are tabulated in Table 7.

The image classification of the pre-COVID and postCOVID image through different object-based image classification techniques visually presents the change in the study area. It has been observed that the pre-COVID image "MLC scheme" has shown the most satisfactory results both visually and numerically. In the post, COVID image classification "MLC scheme" has produced the most reliable results. As in both cases, image classification is performed through MLC technique, and most of the classes in image classification have appeared. In PC technique building, trees and roads classes are most dominant while other classes remain inactive during the classification. In the pre-COVID classified image through MDC technique, four classes are prevalent, i.e., trees, cars, roads, and buildings.

In contrast, three classes are dominant in the postCOVID classified image, i.e., trees, roads, and buildings. In the pre-classified image through SAM technique, four classes are most prevalent, i.e., building, grassland, trees, and road. In contrast, only three classes are dominant in the post-classified image, i.e., building, tree, and road. SID classification technique has produced the most abrupt result against the image classification. In the pre-COVID image, it is observed that the class "car" is most dominant, whereas, in the post-COVID image, the class building is dominant. This classification scheme has produced the worst classification results. Finally, through SVM classification scheme, buildings, trees, and roads are the most prevalent class in the pre-COVID classified image. In the post-COVID image tree, cars and roads have appeared as the most dominant image class.

The linear fitting of the degree (1) corresponding to kappa coefficient $\left(K_{p}\right)$ and overall accuracy (OA) is present in Fig. 23. The curve fitting value is $R^{2} \geq 0.99$ for pre-COVID and post-COVID images. This suggests that the overall classification accuracy is directly proportional to the $K_{p}$. One important conclusion derived from this experiment is that MLC has emerged as a reasonable classification scheme with superior accuracy and kappa coefficient.

\section{Discussion}

In this research work, an innovative combination of PBCD and OBCD techniques is presented. GLCM has emerged as a creative technique that contains information about the "statistical and spectral arrangement" of the image pixels. This information can be concluded in common words as GLCM offer information about the changes occurring inside the image, i.e., information about the spectral and spatial arrangement of the image pixels. The OBCD techniques have emerged as an ideal method to represent the changes developed in the study area visually. Here, users can create an object (class) of interest and compare pre-and post-image specifications based on the same object. Through OBCD technique, not only classes can be made, but the accuracy of the classification can also be obtained. Thus it is observed 
Table 7 Classification features for the post-COVID classified image

\begin{tabular}{|c|c|c|c|c|c|c|c|}
\hline \multirow[t]{2}{*}{ Scheme } & \multirow[t]{2}{*}{ Classification class } & \multicolumn{4}{|c|}{ Classification features } & \multirow[t]{2}{*}{ Overall accuracy } & \multirow[t]{2}{*}{ Kappa coefficient } \\
\hline & & UA & PA & OE & $\mathrm{CE}$ & & \\
\hline \multirow[t]{6}{*}{ Parallelepiped classification } & Building & 25.96 & 99.45 & 0.55 & 74.04 & \multirow[t]{6}{*}{$(4350 / 7727)=56.2961 \%$} & \multirow[t]{6}{*}{0.4749} \\
\hline & Tree & 98.09 & 99.55 & 0.45 & 1.91 & & \\
\hline & Road & 86.29 & 99.39 & 0.61 & 13.71 & & \\
\hline & Grassland & 0.00 & 0.00 & 100.00 & 100.00 & & \\
\hline & Metro & 100.00 & 16.60 & 83.40 & 0.00 & & \\
\hline & Car & 0.00 & 0.00 & 100.00 & 100.00 & & \\
\hline \multirow[t]{6}{*}{ Minimum distance classification } & Building & 66.45 & 76.84 & 23.16 & 33.55 & \multirow[t]{6}{*}{$(5189 / 7727)=67.1541 \%$} & \multirow[t]{6}{*}{0.6013} \\
\hline & Tree & 99.90 & 78.15 & 21.85 & 0.10 & & \\
\hline & Road & 72.97 & 99.94 & 0.06 & 27.03 & & \\
\hline & Grassland & 93.21 & 80.86 & 19.14 & 06.79 & & \\
\hline & Metro & 31.73 & 12.04 & 87.96 & 68.27 & & \\
\hline & Car & 0.75 & 33.33 & 66.67 & 99.25 & & \\
\hline \multirow[t]{6}{*}{ Maximum likelihood classification } & Building & 66.30 & 77.57 & 22.43 & 33.70 & \multirow[t]{6}{*}{$(5691 / 7727)=73.6508 \%$} & \multirow[t]{6}{*}{0.6746} \\
\hline & Tree & 96.71 & 98.66 & 1.34 & 3.29 & & \\
\hline & Road & 85.25 & 99.02 & 0.98 & 14.75 & & \\
\hline & Grassland & 81.07 & 78.11 & 21.98 & 18.93 & & \\
\hline & Metro & 48.53 & 27.51 & 72.49 & 51.47 & & \\
\hline & Car & 0.48 & 9.52 & 90.48 & 99.52 & & \\
\hline \multirow[t]{6}{*}{ Spectral angle mapper } & Building & 45.84 & 23.45 & 76.55 & 54.16 & \multirow[t]{6}{*}{$(3479 / 7727)=45.0239 \%$} & \multirow[t]{6}{*}{0.3298} \\
\hline & Tree & 89.47 & 36.44 & 63.56 & 10.53 & & \\
\hline & Road & 68.54 & 45.84 & 54.16 & 31.46 & & \\
\hline & Grassland & 54.45 & 36.54 & 63.46 & 45.55 & & \\
\hline & Metro & 78.84 & 28.54 & 71.46 & 21.16 & & \\
\hline & Car & 68.54 & 34.45 & 65.55 & 31.46 & & \\
\hline \multirow[t]{6}{*}{ Spectral information divergence } & Building & 26.95 & 60.48 & 39.52 & 73.05 & \multirow[t]{6}{*}{$(2260 / 7727)=29.2481 \%$} & \multirow[t]{6}{*}{0.1735} \\
\hline & Tree & 94.11 & 63.16 & 36.84 & 5.89 & & \\
\hline & Road & 8.05 & 3.86 & 96.14 & 91.95 & & \\
\hline & Grassland & 33.94 & 35.63 & 64.37 & 66.06 & & \\
\hline & Metro & 81.82 & 2.42 & 97.58 & 18.18 & & \\
\hline & Car & 1.48 & 57.14 & 42.86 & 98.52 & & \\
\hline \multirow[t]{6}{*}{ Support vector machine } & Building & 31.49 & 93.47 & 6.53 & 68.51 & \multirow[t]{6}{*}{ (2758/7727) $35.6930 \%$} & \multirow[t]{6}{*}{0.2285} \\
\hline & Tree & 41.15 & 96.87 & 3.13 & 58.85 & & \\
\hline & Road & 32.99 & 27.05 & 72.95 & 67.01 & & \\
\hline & Grassland & 0.00 & 0.00 & 100.00 & 0.00 & & \\
\hline & Metro & 0.00 & 0.00 & 100.00 & 0.00 & & \\
\hline & Car & 0.00 & 0.00 & 100.00 & 0.00 & & \\
\hline
\end{tabular}

that PBCD GLCM approach and OBCD both techniques provide useful information related to image classification. Usage of any one of the techniques or both techniques depends upon the application only. In this research, a fusion methodology of PBCD and OBCD techniques is presented to extract maximum information from the study area. Finally, based on the experimental results, a model is developed to exact the full report of the study area shown in Fig. 24. It is also expected that the proposed model will work efficiently with other types of images, including multispectral images and hyperspectral images.
The presented model for image analysis includes PBCD based GLCM approach, which provides complete image information about the "spectral and spatial arrangement." In OBCD process, select the best classification methodology having superior accuracy with maximum $K p$. Thus all the features obtained from GLCM and OBCD assist to under an event altogether.

The texture classification of the study area presented in this work presents information about specific changes caused due to COVID lockdown in the study area. These changes are represented in the histogram signature plot of the pre-COVID 
Fig. 23 Relationship of degree (1) between kappa coefficient and overall accuracy. a PreCOVID. b Post-COVID

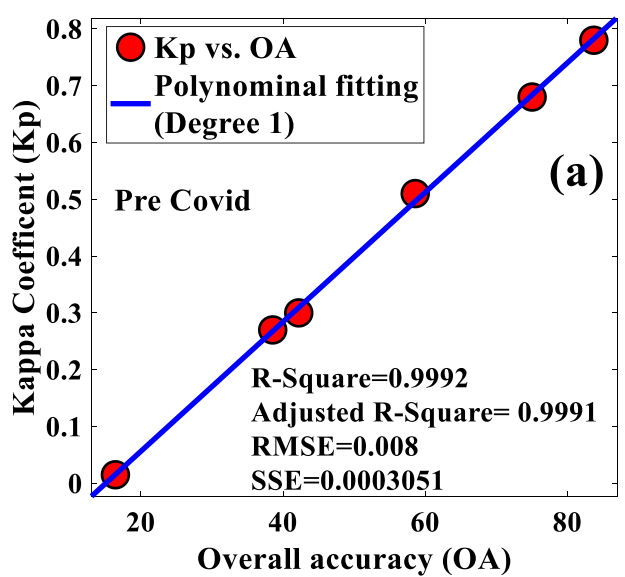

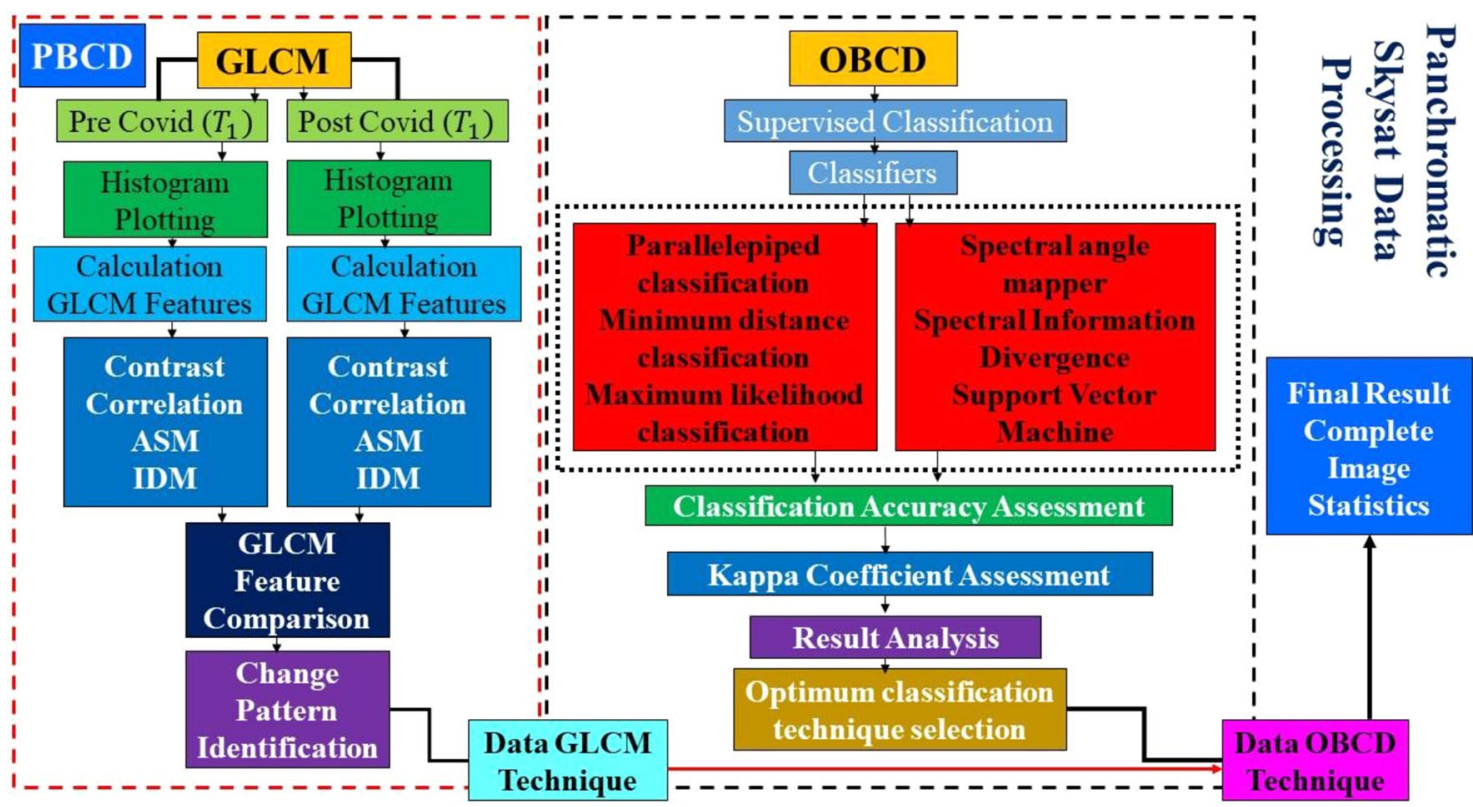

Fig. 24 Proposed PBCD and OBCD fusion model

and post-COVID images. GLCM features have also presented a pattern of statistical variation in which ASM have show exponential increase in the feature values whereas correlation, energy, and IDM have shown fall in their feature values. The role of OBCD technique in change identification is remarkably selecting an appropriate classification algorithm depends upon several factors like the study area. If the spatial resolution of the image is less (study area has less number of image pixels), then the user has a clear view of the classification classes. Then, in this case, good classification accuracy is expected from SVM, ML, MLC approaches. Likewise, the $K_{p}$ will also have a high value. Now, suppose the study area has high resolution with a large number of image pixels. In this case, it is expected that selecting an appropriate number of pixel numbers for a particular class is difficult. Thus low overall classification accuracy along with less $K_{p}$ will be obtained in this case. OBCD technique is quite effective in representing the visual difference of the developed changes. All the classified objects are visible and easily distinguishable through OBCD schemes. Thus the fusion of GLCM and OBCD techniques are desirable for the cases where internal and external information of the study area is required.

\section{Conclusion}

This research work presents two different methodologies fused to obtain maximum information from data of interest. Firstly quantification of texture features based on the "grey level co-occurrence matrix (GLCM)" technique is performed. In the second step, image classification based on "object-based change detection (OBCD)" methods visually 
represents the transformation developed in the study area due to COVID lockdown. Pre-COVID and post-COVID (during lockdown) panchromatic images of Connaught Place, New Delhi, are analyzed in this research work to develop an accurate model for the study area. Texture classification of the images is performed based on visual texture features for eight distances and four orientations. Six different image classification methodologies are used for performing the image classification of the study area. These methodologies are "Parallelepiped classification (PC)," "Minimum distance classification (MDC)," "Maximum likelihood classification (MLC)," "Spectral angle mapper (SAM)," "Spectral Information Divergence (SID)" and "Support Vector Machine (SVM)." GLCM features quantification has provided a novel pattern of texture variations, i.e., contrast, correlation, ASM, and IDM. OBCD based MLC techniques have provided a maximum classification accuracy of $83.68 \%$ and $73.65 \%$ for the pre-COVID and post-COVID image data. Finally, a model is presented based on the above investigation for analyzing before and after COVID images. The model follows a two-step methodology with a final fusion of the obtained information to produce complete information about the study area numerically and visually.

Acknowledgements The authors are thankful to all anonymous reviewers and Editor-in-Chief for their comments, concerns, queries, and constructive suggestions. The authors are also grateful to "Planet." for the panchromatic Skysat pre- and post-COVID imagery used in this research work.

\section{References}

Agariga F, Abugre S, Appiah M (2021) Spatio-temporal changes in land use and forest cover in the Asutifi North District of Ahafo Region of Ghana (1986-2020). Environ Chall 5:100209. https:// doi.org/10.1016/j.envc.2021.100209

Annett I (2010) UK Space Agency (Government of UK) Retrieved 26 Jul 2021. https://www.gov.uk/government/organisations/ uk-space-agency

Arefin R, Meshram SG, Santos CA, Silva RM, Pushparaj J (2020) Hybrid modelling approach for water body change detection at Chalan Beel area in northern Bangladesh. Environ Earth Sci 79:442. https://doi.org/10.1007/s12665-020-09185-y

Atangana A, Araz Sİ (2020) Mathematical model of COVID-19 spread in Turkey and South Africa: theory, methods, and applications. Adv Differ Equ 2020:659. https://doi.org/10.1186/ s13662-020-03095-w

Baker K, Culton E, Lang J, Lewis Z, Perez-Alemany R, Rizzo A (2020) An updated re-entry analysis of the Hubble Space Telescope. J Space Saf Eng 7(3):404-410

Caballero GR, Platzeck G, Pezzola A, Casella A, Winschel C, Silva SS (2020) Assessment of multi-date Sentinel-1 polarizations and GLCM texture features capacity for onion and sunflower classification in an irrigated valley: an object level approach. Agronomy 10(6):845

Calhoun D (1916) Boeing (Boeing: Aerospace company). Retrieved 26 Jul 2021. https://www.boeing.com/
Chandrashekar S (2016) Space, war, and deterrence: a strategy for India. Int J Space Politics Policy 14(2-3):135-157

Chen G, Weng Q, Hay GJ, He Y (2018) Geographic object-based image analysis (GEOBIA): emerging trends and future opportunities. Gisci Remote Sens 55(2):159-182

Cohen IJ, Rymer AM (2020) Cross-NASA divisional relevance of an Ice Giant mission. Trans R Soc Am 378(2187):1-12

d'Italie R (2020) Drones and the coronavirus: do these applications make sense? (We Robotics). Retrieved 10 Mar 2021. https:// blog.werobotics.org/2020/04/09/drones-coronavirus-no-sense/

Damle SV, Daniel RR, Lavakare PJ (1976) Energetic neutrons and gamma rays measured on the Aryabhata satellite. Pramana 7:355-368. https://doi.org/10.1007/BF02847032

Du P, Wang X, Chen D, Liu S, Lin C, Meng Y (2020) An improved change detection approach using tri-temporal logic-verified change vector analysis. ISPRS J Photogramm Remote Sens 161:278-293. https://doi.org/10.1016/j.isprsjprs.2020.01.026

Dunbar B (1958) About NASA (National Aeronautics and Space Administration). Retrieved 26 Jul 2021. https://www.nasa.gov/

Eid AN, Olatubara CO, Ewemoje TA, El-Hennawy MT, Farouk $\mathrm{H}$ (2020) Inland wetland time-series digital change detection based on SAVI and NDWI indices: Wadi El-Rayan lakes, Egypt. Remote Sens Appl: Soc Environ 19:100347. https://doi.org/10. 1016/j.rsase. 2020.100347

François-Philippe (1989) Canadian Space Agency (Governement of Canada). Retrieved 26 Jul 2021. https://www.asc-csa.gc.ca/eng/ Default.asp

Garg M, Dhiman G (2021) A novel content-based image retrieval approach for classification using GLCM features and texture fused LBP variant neural computing and applications. Neural Comput Appl 33:1311-1328. https://doi.org/10.1007/ s00521-020-05017-z

Gotlieb CC, Kreyszig HE (1990) Texture descriptors based on co-occurrence matrices. Comput vis Graph Image Process 51(1):70-86

Government R (1992) ROSCOSMOS (State Space Corporation ROSCOSMOS). Retrieved 26 Jul 2021. http://en.roscosmos.ru/

Haider SA, Pandya BM (2015) Probing of meteor showers at Mars during the encounter of comet $\mathrm{C} / 2013 \mathrm{~A} 1$ : predictions for the arrival of MAVEN/Mangalyaan. Geosci Lett 2:8. https://doi.org/ 10.1186/s40562-015-0023-2

Hajeb M, Karimzadeh S, Fallahi A (2020) Seismic damage assessment in Sarpole-Zahab town (Iran) using synthetic aperture radar (SAR) images and texture analysis. Nat Hazards 103:347-366. https:// doi.org/10.1007/s11069-020-03991-0

Haralick RM, Shanmugam K, Dinstein I (1973) Textural features for image classification. IEEE Trans Syst Man Cybern SMC-3(6):610-621

Hazarika N, Jain VK, Srivastava A (2015) Source identification and metallic profiles of size-segregated particulate matters at various sites in Delhi. Environ Monit Assess 187:602. https://doi.org/10. 1007/s10661-015-4809-7

Hunt ER Jr (2021) Spectral discrimination using infinite leaf reflectance and simulated canopy reflectance. Int J Remote Sens 42(8):3039-3055

Hussain M, Chen D, Cheng A, Wei H, Stanley D (2013) Change detection from remotely sensed images: from pixel-based to objectbased approaches. ISPRS J Photogramm Remote Sens 80:91-106

Iqbal N, Mumtaz R, Shafi U, Zaidi SM (2021) Gray level co-occurrence matrix (GLCM) texture-based crop classification using low altitude remote sensing platforms. PeerJ Comput Sci 7:e536. https:// doi.org/10.7717/peerj-cs.536

Jiang S, Jiang C, Jiang W (2020) Efficient structure from motion for large-scale UAV images: a review and a comparison of SfM tools. ISPRS J Photogramm Remote Sens 167:230-251. https://doi.org/ 10.1016/j.isprsjprs.2020.04.016 
Jorden MA, Rudman SL, Villarino E, Hoferka S et al (2020) Evidence for limited early spread of COVID-19 Within the United States, January-February 2020. MMWR Morb Mortal Wkly Rep 69(22):680-684

Kalaiselvi S, Gomathi V (2020) Alpha-cut induced fuzzy deep neural network for change detection of SAR images. Appl Soft Comput 95:106510. https://doi.org/10.1016/j.asoc.2020.106510

Kejian Z (1993) China National Space Administration (Government of China). Retrieved 26 Jul 2021. http://www.cnsa.gov.cn/english/

Kerle N, Nex F, Gerke M, Duarte D, Vetrivel A (2020) UAV-based structural damage mapping: a review. ISPRS Int J Geo-Inf 9(1):14

Khelifi L, Mignotte M (2020) Deep learning for change detection in remote sensing images: comprehensive review and meta-analysis. IEEE Access 8:126385-126400. https://doi.org/10.1109/ ACCESS.2020.3008036

Kundu K, Halder P, Mandal JK (2021) Detection and prediction of sundarban reserve forest using the CA-Markov chain model and remote sensing data. Earth Sci Inf 1:1-18. https://doi.org/10.1007/ s12145-021-00648-9

Leonga WC, Bahadori A, Zhang J, Ahmad Z (2021) Prediction of water quality index (WQI) using support vector machine (SVM) and least square-support vector machine (LS-SVM). Int J River Basin Manag 19(2):149-156

Li B, Yang Z-P, Chen D-Q, Liang S-Y, Ma H (2020) Maneuvering target tracking of UAV based on MN-DDPG and transfer learning. Def Technol. https://doi.org/10.1016/j.dt.2020.11.014

Liu R, Wang R, Huang J, Li J, Jiao L (2020) Change detection in SAR images using multiobjective optimization and ensemble strategy. IEEE Geosci Remote Sens Lett. https://doi.org/10.1109/LGRS. 2020.3005140

Maimaitijiang M, Sagan V, Sidike P, Daloye AM, Erkbol H, Fritschi FB (2020) Crop monitoring using satellite/UAV data fusion and machine learning. Remote Sens 12(9):1357

Marshall W, Boshuizen C, Schingler R (2010) Planet (Planet Labs Inc). Retrieved 26 Jul 2021. https://www.planet.com/

Mathieu P-P, Borgeaud M, Desnos Y-L, Rast M, Brockmann C, See L (2017) The ESA's earth observation open science program [space agencies]. IEEE Geosci Remote Sens Mag 5(2):86-96

Mukherjee T, Asutosh A, Pandey SK, Yang L, Gogoi PP, Panwar A, Vinoj V (2020) Increasing potential for air pollution over megacity New Delhi: a study based on 2016 Diwali episode. Aerosol Air Qual Res 18(9):2510-2518

Muraleedharan D, Haridasan P, Samad AA, Varier GS, Vinod P (2019) Reconfigurable wavelet-based real-time imaging system for low bit rate telemetry space missions. IEEE Aerosp Electron Syst Mag 34(5):22-29

Musk E (2002) TRANSPORTER-2 MISSION (SpaceX). Retrieved 26 Jul 2021. https://www.spacex.com/

Nascimento IJ, Cacic N, Abdulazeem HM, Groote TC, Jayarajah U (2020) Novel coronavirus infection (COVID-19) in humans: a scoping review and meta-analysis. J Clin Med 9(4):941

Nie L, Sun W, Wang S, Ning Z, Rodrigues JJ, Wu Y, Li S (2021) Intrusion detection in green internet of things: a deep deterministic policy gradient-based algorithm. IEEE Trans Green Commun Netw 5(2):778-788

Niethammer U, James MR, Rothmund S, Travelletti J, Joswig M (2012) UAV-based remote sensing of the Super-Sauze landslide: evaluation and results. Eng Geol 128:2-11. https://doi.org/10.1016/j. enggeo.2011.03.012

Otto A, Agatz N, Campbell J, Golden B, Pesch E (2018) Optimization approaches for civil applications of unmanned aerial vehicles (UAVs) or aerial drones: a survey. Network 72(4):411-458

Palermo E (2018) Australian Space Agency (Australian Government). Retrieved 26 Jul 2021. www.industry.gov.au
Papanastassiou DA, Wasserburg GJ (1971) Rb-Sr ages of igneous rocks from the Apollo 14 mission and the age of the Fra Mauro formation. Earth Planet Sci Lett 12(1):36-48

Pati C, Panda KA, Tripathy AK, Pradhan KS, Patnaik S (2020) A novel hybrid machine learning approach for change detection in remote sensing images. Eng Sci Technol 23(5):973-981

Polykretis C, Grillakis MG, Alexakis DD (2020) Exploring the impact of various spectral indices on land cover change detection using change vector analysis: a case study of Crete Island, Greece. Remote Sens 12(2):319

Rimal B, Rijal S, Kunwar R (2020) Comparing support vector machines and maximum likelihood classifiers for mapping of urbanization. J Indian Soc Remote Sens 48:71-79. https://doi.org/10.1007/ s12524-019-01056-9

Saha S, Bovolo F, Bruzzone L (2021) Building change detection in VHR SAR images via unsupervised deep transcoding. IEEE Trans Geosci Remote Sens 59(3):1917-1929

Sarabhai V (1969) Indian Space Research Organisation (Department of Space, Indian Space Research Organisation). Retrieved 26 Jul 2021. https://www.isro.gov.in/

Schwartz C, Ramos LP, Duarte LT, Pinho MD, Pettersson MI (2020) Change detection in UWB SAR images based on robust principal component analysis. Remote Sens 12(12):1-11

Seydi ST, Hasanlou M, Amani M (2020) A new end-to-end multidimensional CNN framework for land cover/land use change detection in multi-source remote sensing datasets. Remote Sens 12(12): $1-38$

Shi W, Zhang M, Zhang R, Chen S, Zhan Z (2020) Change detection based on artificial intelligence: state-of-the-art and challenges. Remote Sens 12(10): 1688

Shukla K, Kumar P, Mann S, G., \& Khare, M. (2020) Mapping spatial distribution of particulate matter using kriging and inverse distance weighting at supersites of megacity Delhi. Sustain Cities Soc 54:101997. https://doi.org/10.1016/j.scs.2019.101997

Sierra Nevada Corporation (1963) Dream, innovate, inspire (Sierra Nevada Corporation). Retrieved 26 Jul 2021. https://www.sncorp. $\mathrm{com} /$

Singh US, Singh RK (2020) Application of maximum-likelihood classification for segregation between Arctic multi-year ice and firstyear ice using SCATSAT-1 data. Remote Sens Appl: Soc Environ 18:100310. https://doi.org/10.1016/j.rsase.2020.100310

Soni PK, Rajpal N, Mehta R, Mishra VK (2021) Urban land cover and land use classification using multispectral Sentinal-2 imagery. Multimed Tools Appl 1211:1-15. https://doi.org/10.1007/ s11042-021-10991-0

Sotin C, Kalousová K, Tobie G (2021) Titan's interior structure and dynamics after the Cassini-Huygens mission. Annu Rev Earth Planet Sci 49:579-607. https://doi.org/10.1146/annur ev-earth-072920-052847

Thompson DW (1982) Surviving Space (Orbital Sciences Corporation). Retrieved $26 \mathrm{Jul}$ 2021. https://www.northropgrumman.com/

Tiwari A, Arun G, Vishwakarma BD (2021) Parameter importance assessment improves the efficacy of machine learning methods for predicting snow avalanche sites in Leh-Manali Highway, India. Sci Total Environ 794:148738. https://doi.org/10.1016/j.scitotenv. 2021.148738

Tomsick JA, Coughenour BM, Hare J, Krivonos R, Bodaghee A (2021) Using Chandra localizations and Gaia distances and proper motions to classify hard X-ray sources discovered by INTEGRAL. Astrophys J 914(48):1-17

Tong X, Feng Y (2020) A review of assessment methods for cellular automata models of land-use change and urban growth. Int J Geogr Inf Sci 34:866-898. https://doi.org/10.1080/13658816. 2019.1684499 
Venugopal N (2020) Automatic semantic segmentation with DeepLab dilated learning network for change detection in remote sensing images. Neural Process Lett 51:2355-2377. https://doi.org/10. 1007/s11063-019-10174-x

Wang L, Shen Y, Li M, Chuang H, Ye Y, Zhao H, Wang H (2020a) Clinical manifestations and evidence of neurological involvement in 2019 novel coronavirus SARS-CoV-2: a systematic review and meta-analysis. J Neurol 267:2777-2789. https://doi.org/10.1007/ s00415-020-09974-2

Wang M, Tan K, Jia X, Wang X, Chen Y (2020b) A deep Siamese network with hybrid convolutional feature extraction module for change detection based on multi-sensor remote sensing images. Remote Sens 12(2):1-18

Wang SW, Gebru BM, Lamchin M, Kayastha RB, Lee W-K (2020c) Land use and land cover change detection and prediction in the Kathmandu District of Nepal using remote sensing and GIS. Sustainability 12(9):3925

Wang M, Huang Z, Zhang X, Zhang Y, Chen M (2021) Altered mineral mapping based on ground-airborne hyperspectral data and wavelet spectral angle mapper tri-training model: case studies from Dehua-Youxi-Yongtai Ore District, Central Fujian, China. Int J Appl Earth Obs Geoinf 102:102409. https://doi.org/10.1016/j. jag.2021.102409

Woodcock CE, Loveland TR, Herold M, Bauer ME (2020) Transitioning from change detection to monitoring with remote sensing: a paradigm shift. Remote Sens Environ 238:111558. https://doi.org/ 10.1016/j.rse.2019.111558
Wörner J-D (1975) European Robotic Arm is launched into space (European Space Agency). Retrieved 26 July 2021. https://www. esa.int/

Xu H, Yan C, Fu Q, Xiao K, Yu Y, Han D, Wang W (2020) Possible environmental effects on the spread of COVID-19 in China. Sci Total Environ 731:139211. https://doi.org/10.1016/j.scitotenv. 2020.139211

Yamakawa H (2003) Japan Aerospace Exploration Agency (JAXA). Retrieved 26 Jul 2021. https://global.jaxa.jp/

Zhang Y, Peng D, Huang X (2018) Object-based change detection for VHR images based on multiscale uncertainty analysis. IEEE Geosci Remote Sens Lett 15(1):13-17

Zhang C, Yue P, Tapete D, Jiang L, Shangguan B (2020) A deeply supervised image fusion network for change detection in high resolution bi-temporal remote sensing images. ISPRS J Photogramm Remote Sens 166:183-200. https://doi.org/10.1016/j.isprs jprs.2020.06.003

Zhao R, Peng G-H, Yan W-D, Pan L-L, Wang L-Y (2021) Change detection in SAR images based on superpixel segmentation and image regression. Earth Sci Inf 14:69-79. https://doi.org/10.1007/ s12145-020-00532-y

Zhu Y, Jia Z, Yang J, Kasabov NK (2020) Change detection in multitemporal monitoring images under low illumination. IEEE Access 8:126700-126712. https://doi.org/10.1109/ACCESS.2020.3008262

Publisher's Note Springer Nature remains neutral with regard to jurisdictional claims in published maps and institutional affiliations. 